\title{
Transcriptional analysis of an immune-responsive serine protease from Indian malarial vector, Anopheles culicifacies Janneth Rodrigues $^{\dagger 1,4}$, Neema Agrawal ${ }^{\dagger 1}$, Anil Sharma ${ }^{\dagger 1,3}$, Pawan Malhotra ${ }^{1,2}$, Tridibes Adak ${ }^{1,3}$, Virander S Chauhan ${ }^{1,2}$ and Raj K Bhatnagar*1
}

\begin{abstract}
Address: ${ }^{1}$ Insect Resistance Group, International Centre for Genetic Engineering and Biotechnology (ICGEB) PO Box 10504, Aruna Asaf Ali Marg, New Delhi 110067, India, ${ }^{2}$ Malaria Group, ICGEB, New Delhi 67, India, ${ }^{3}$ National Institute of Malaria Research 2, Nanak Enclave, (Radio Colony), Delhi 110009, India and ${ }^{4}$ Laboratory of Malaria and Vector Research, National Institute of Allergy and Infectious Diseases, NIH, Bethesda, Maryland, USA

Email: Janneth Rodrigues - rodriguesj@niaid.nih.gov; Neema Agrawal - neema@icgeb.res.in; Anil Sharma - anil.mrc@gmail.com; Pawan Malhotra - pawan@icgeb.res.in; Tridibes Adak - adak.mrc@gmail.com; Virander S Chauhan - virander@icgeb.res.in; Raj K Bhatnagar* - raj@icgeb.res.in

* Corresponding author †Equal contributors
\end{abstract}

Published: 15 May 2007

BMC Molecular Biology 2007, 8:33 doi:10.1186/147I-2199-8-33

This article is available from: http://www.biomedcentral.com/I47/-2199/8/33

(C) 2007 Rodrigues et al; licensee BioMed Central Ltd.

This is an Open Access article distributed under the terms of the Creative Commons Attribution License (http://creativecommons.org/licenses/by/2.0), which permits unrestricted use, distribution, and reproduction in any medium, provided the original work is properly cited.

\begin{abstract}
Background: The main vector for transmission of malaria in India is the Anopheles culicifacies mosquito species, a naturally selected subgroup of which is completely refractory (R) to transmission of the malaria parasite, Plasmodium vivax;
\end{abstract}

Results: Here, we report the molecular characterization of a serine protease (acsp30)-encoding gene from A. culicifacies, which was expressed in high abundance in the refractory strain compared to the susceptible (S) strain. The transcriptional upregulation of acsp30 upon Plasmodium challenge in the refractory strain coincided with ookinete invasion of mosquito midgut. Gene organization and primary sequence of acsp30 were identical in the R and $\mathrm{S}$ strains suggesting a divergent regulatory status of acsp30 in these strains. To examine this further, the upstream regulatory sequences of acsp30 were isolated, cloned and evaluated for the presence of promoter activity. The 702 bp upstream region of acsp30 from the two strains revealed sequence divergence. The promoter activity measured by luciferase-based reporter assay was shown to be I.5-fold higher in the $\mathrm{R}$ strain than in the $\mathrm{S}$. Gel shift experiments demonstrated a differential recruitment of nuclear proteins to upstream sequences of acsp30 as well as a difference in the composition of nuclear proteins in the two strains, both of which might contribute to the relative abundance of acsp30 in the R strain;

Conclusion: The specific upregulation of acsp30 in the $\mathrm{R}$ strain only in response to Plasmodium infection is suggestive of its role in contributing the refractory phenotype to the $A$. culicifacies mosquito population.

\section{Background}

The Anopheles culicifacies mosquito is the main vector of the human malaria parasite, $P$. vivax, in the Indian sub- continent and is responsible for approximately $65 \%$ of new malaria cases annually [1]. The natural transmission cycle of Plasmodium parasite requires successful comple- 
tion of a complex sporogonic cycle in the midgut and the salivary glands of the Anopheles mosquito, a process that takes place over a period of two weeks. This developmental cycle can be blocked by the innate immune responses of the mosquito thereby resulting in the elimination of pathogen in the vector itself [2].

Mosquito vectors differ in their efficiency of transmitting malaria; some are refractory and completely block transmission of parasite [2]. Naturally evolved and genetically selected refractory strains are important for the study of mechanisms that mediate Plasmodium killing [3-5]. Genetically selected susceptible and refractory strains (4Ar/r and L3-5, respectively) are described in African mosquito Anopheles gambiae [3]. The R strain blocks parasite development by melanotically encapsulating the ookinetes after invasion of the midgut [6]. Understanding the molecular basis of such refractory phenotypes may contribute to the development of novel strategies for malaria control, which would rely on elimination of the parasite in the mosquito itself.

Recently, Adak et al. (2006) reported the isolation of a naturally occurring field strain of A. culicifacies that is $100 \%$ refractory to $P$. vivax and partially resistant to $P$. falciparum and $P$. vinckei (rodent parasite) [7,8]. In the $\mathrm{R}$ strain, brown capsular ookinetes characteristic of the melanization reaction were microscopically observed in the mosquito midgut within 24 hours of Plasmodium - infected blood feeding. Normal uncoated ookinetes were observed in the $S$ strain indicating that strain refractoriness in $A$. culicifacies could be attributed to melanotic encapsulation of ookinetes. The melanotic capsule consists of a proteinaceous poly-quinone material surrounding the parasite and killing of the parasite may be mediated by toxic byproducts of the melanization cascade reactions, such as free radicals or by starvation within the capsule $[9,10]$.

The melanization reaction starts with a modulatory serine protease cascade, leading to proteolytic cleavage and activation of prophenoloxidases (PPOs) to phenoloxidases (POs), the key enzymes for melanin production $[11,12]$. In addition, serine proteases are also involved in signaling and amplification cascades that lead to the activation of specific defense mechanisms, such as melanization, coagulation and induction of anti-microbial peptides. An increasing number of serine proteases involved in immune responses have been isolated and characterized from A. gambiae. The majority of these serine proteases are present in the hemolymph and expressed by hemocytes. In A. gambiae, CLIPB9, CLIPB4 and CLIPB1 serine proteases are induced during immune responses and have sequences characteristic of prophenoloxidase activating enzymes [13-17]. The role of several of these proteases in melanization and killing of parasites has been examined by RNA interference [18-20].

In the present study, we report the isolation of a serine protease (acsp30) from the body tissue of A. culicifacies. Acsp30 was poorly transcribed in the $S$ strain whereas abundant transcript was observed in the R strain. Expression of acsp30 was constitutive in both the strains but was strongly upregulated upon parasite invasion in the $\mathrm{R}$ strain. Although, the nucleotide sequence of acsp30 cDNA and genomic clone were identical in $\mathrm{R}$ and $\mathrm{S}$ strains, a significant sequence divergence of upstream regions was observed. The promoter in $\mathrm{R}$ and $\mathrm{S}$ strains was localized within -702 bp of the translation initiation site; quantification of promoter strength by the luciferase-based reporter gene assay revealed a 1.5-fold higher activity in the R strain compared to the S strain. Our studies suggest divergent promoter sequences recruit differential transcription factors resulting in a varied expression of acsp30 in the $\mathrm{R}$ and $\mathrm{S}$ strains. In addition, differential distribution of nuclear proteins in the two strains could also contribute to disparity in acsp30 transcription.

\section{Results \\ Cloning and analysis of acsp30 of A. culicifacies}

A PCR-based strategy was employed to isolate the serine protease-encoding gene (acsp30) from the body tissue (thorax and abdomen) of $A$. culicifacies. The 816 bp fulllength cDNA encoded a 272 amino acid protein, ACSP30, corresponding to molecular mass of approximately 30 $\mathrm{kDa}$. Computer-assisted analysis revealed that the first 17 amino acids of ACSP30 constituted a signal peptide and the next 19 form a propeptide. Mature ACSP30, 236 amino acids in length possessed structural elements characteristic of all serine proteases such as the conserved catalytic triad (HDS) and six cysteine residues (Fig. 1A).

By using the ClustalW alignment program (Mac Vector version 7.0), the deduced amino acid sequence of ACSP30 was aligned with reported sequences from other insects. ACSP30 showed highest sequence similarity (83\%) with a predicted serine protease (Protein Accession: EAA13956.1) from A. gambiae genome (ENSANGP00000014448). Amongst the reported A. gambiae serine proteases, the ACSP30 showed maximum similarity with AgSp24D (31\%) (Fig. 1B) [21]. ACSP30 also shared sequence similarity with serine proteases from Chrysomia bezziana (Screwworm fly) (27\%), Drosophila yakuba (26\%) and Culex quinquefasciatus (30\%) which are reported to function in dissolution of fibrin of blood clots and as proteolytic factors in a variety of processes including embryonic development, tissue remodeling, tumor invasion and inflammation [22]. Interestingly, ACSP30 shared low $(<15 \%)$ sequence homology with the Clip- 


\begin{abstract}
\begin{tabular}{c}
36 \\
MKLF IVVVLACLAAAQAREISYQSILPVRESPRSARIVNGF PAAPGQFPYQVFLSGQTAGGNLACGGSLISA \\
\hline
\end{tabular} $\underset{\triangle}{\text { EWVLTAAHCQVNVIQFTVRAGTTNINEGVVRTSNLIVIHPAYNPSNLNNDIGLIRLSEPVPLGGNIQAVALP }} 144$ AADLSETFLNREATVSGFGRTSDSIPTISTTLNFVHLNI ISNIQCMGTYGAATI IDSTVCAVGRDAANQGTC 216 NGDSGGPLTITENGQSVQIGVVSFVAAVGCEAGFPSGYVRTTHFRDWIRDQSGIKY 272

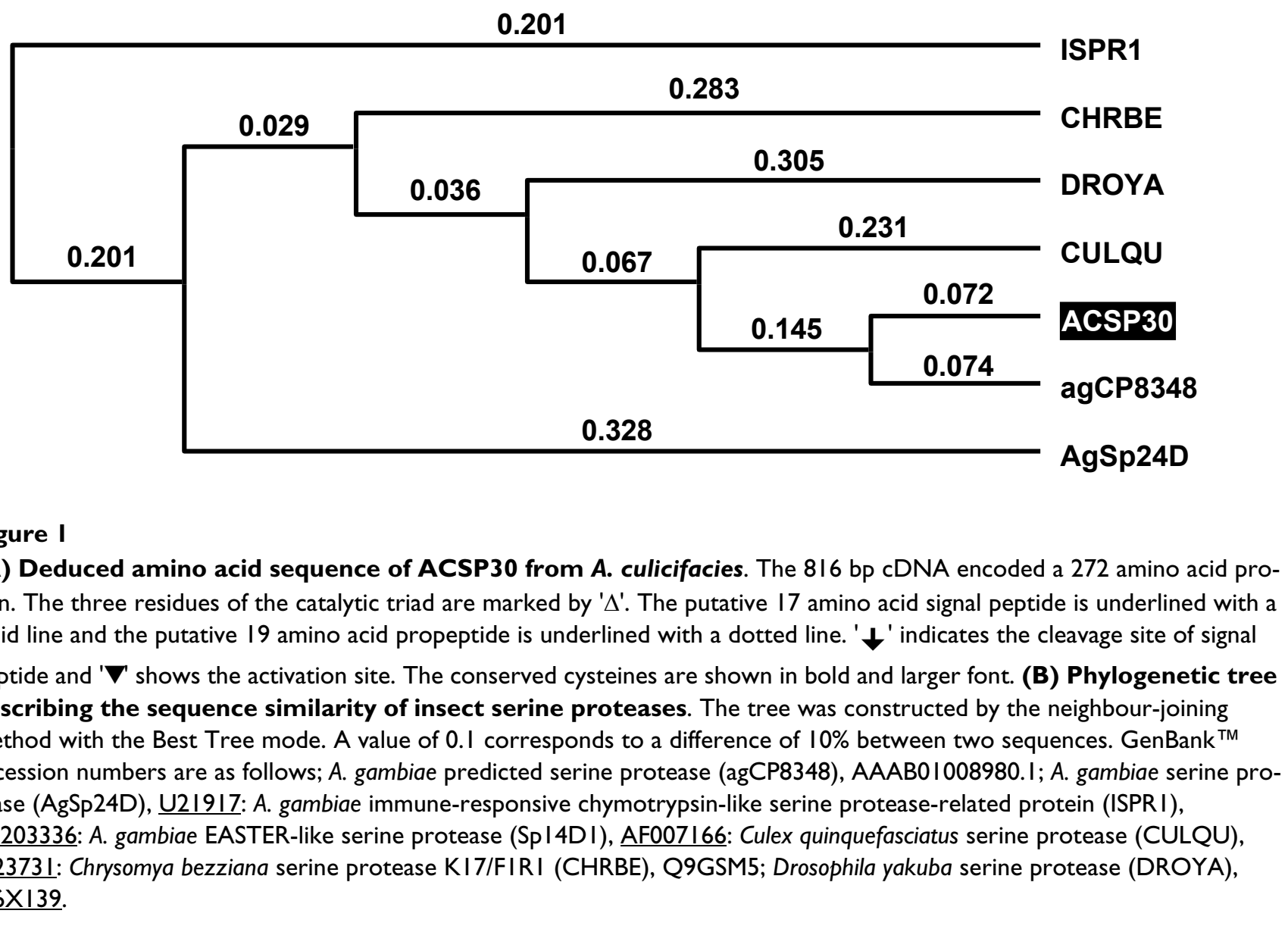

domain serine proteases (CLIPs) that are implicated in defense mechanisms in insects $[18,19,23-26]$.

Differential expression of serine protease in $R$ and $S$ strains The relative expression level of acsp30 transcript in fiveday old naïve adult female mosquitoes of the R and S phenotypes was analyzed by semi-quantitative RT-PCR (Fig. 2A) and real-time PCR (Fig. 2B) using $\beta$-actin as an internal control. Though acsp30 was expressed constitutively in the $\mathrm{R}$ and $\mathrm{S}$ strains, there was a significant difference in their expression levels. In naïve adult female mosquitoes, transcript levels of acsp30 were 40-fold higher in the $\mathrm{R}$ strain compared to S. Acsp30 was expressed constitutively during development of mosquito; a varying pattern of expression was observed from second instar larvae to pupa. A dramatic 300-fold increase in acsp30 transcript was observed in the second instar larvae as compared to the levels present in the adult mosquito (Fig. 2C). 


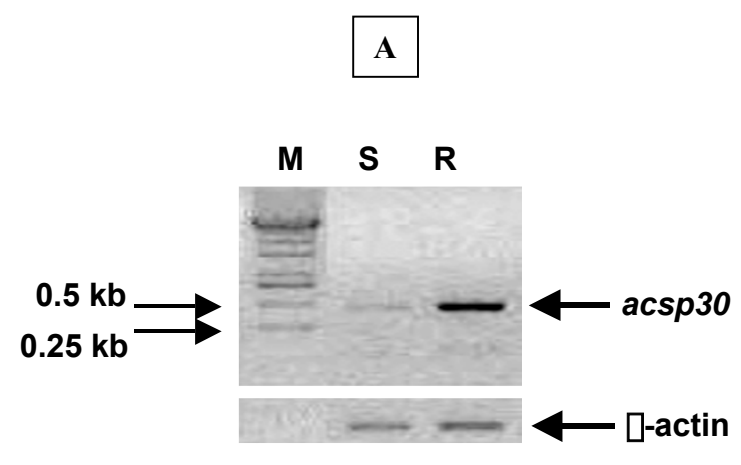

C

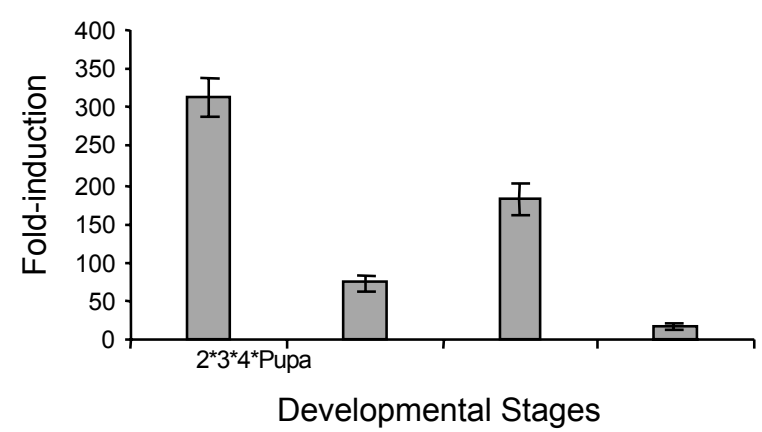

B

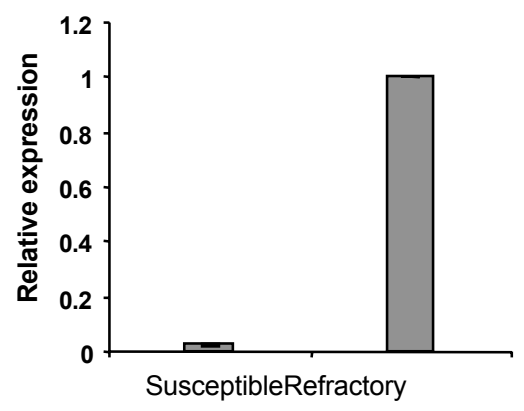

D

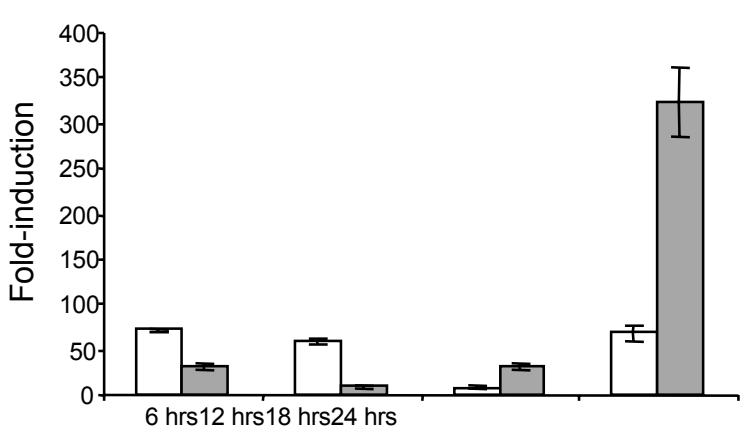

Duration post-bloodfeeding

\section{Figure 2}

Expression pattern of acsp30 in A. culicifacies female mosquito. Determination of relative transcript abundance of ascp30 in R and S strain adult mosquitoes by semi-quantitative RT-PCR (A) and real-time PCR (B). (C) Expression of acsp30 at various stages of mosquito development, second instar $\left(2^{*}\right)$, third instar ( $\left.3^{*}\right)$, fourth instar $\left(4^{*}\right)$ and pupa, is depicted as foldinduction over levels present in naïve adult female mosquitoes by real-time PCR. (D) Temporal induction of acsp30 upon Plasmodium infection. Refractory mosquitoes were fed on uninfected blood (open bars) and on $P$. vinckei infected blood (shaded bars) and transcript levels of acsp30 were measured by real-time PCR at different time intervals post-blood meal (PBM). For all realtime RT-PCR experiments, RNA isolated from body tissue (abdomen and thorax) of mosquitoes was used as template and expression levels of acsp30 were measured by using the Comparative $\mathrm{C}_{\mathrm{T}}$ Method. Transcript levels were normalized to the internal control, $\beta$-actin and shown as fold induction relative to the naïve adult female mosquitoes. Representative data (mean \pm S.D.) from three independent experiments are shown.

\section{Immune responses to Plasmodium infection}

Both refractory and susceptible 4-6 day old adult female mosquitoes were separately fed on blood of Balb/c mice infected with Plasmodium vinckei petteri. The refractory A. culicifacies mosquito strain is partially resistant to the rodent malarial parasite [7]. Mosquitoes fed on blood of uninfected mice were included as blood-fed controls. Temporal expression of acsp30 was monitored by real- time PCR at regular intervals after feeding (Fig. 2D). In the $\mathrm{R}$ strain, a 68-fold increase in acsp30 transcript level was observed 24 hours after feeding on uninfected blood. Mosquitoes fed with infected blood exhibited a 300-fold increase in the transcript levels over the naïve unfed female mosquitoes. The 24 hour post-feeding upregulation of acsp30 expression coincided with microscopic observation of encapsulated Plasmodium ookinetes in the 


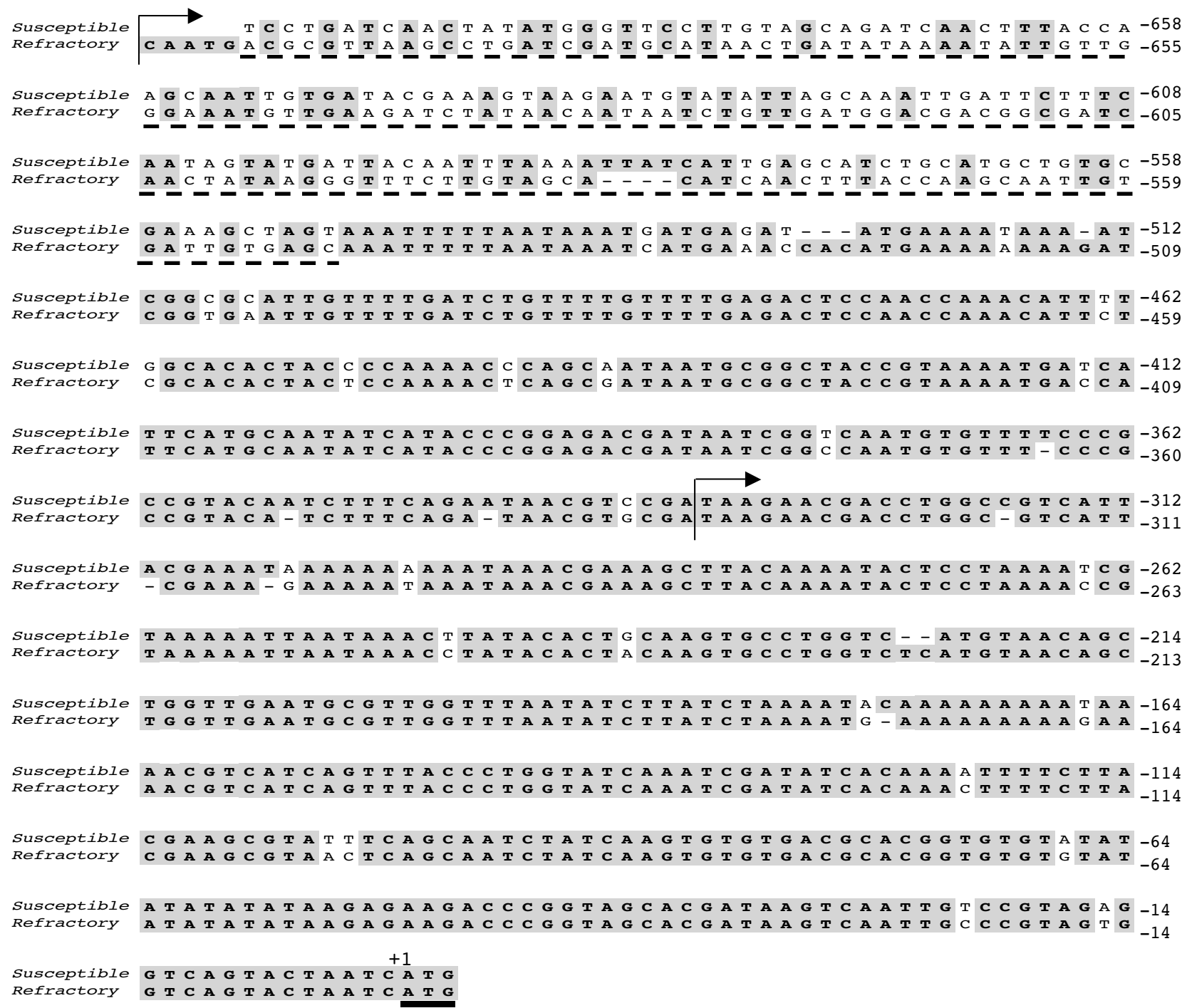

Figure 3

Computer-assisted analysis of acsp30 upstream sequences from refractory and susceptible strains of $A$. culicifacies. (A) Alignment of the two sequences. The translational start site (ATG) is underlined and the numbers to the right denote the positions of the adjacent nucleotide in each line, relative to ATG at $+\mathrm{I}$. The arrows indicate the boundary of the $333 \mathrm{bp}$ and $702 \mathrm{bp}$ constructs used for promoter analysis. The sequence marked with a dashed line represents the region of maximum dissimilarity between $\mathrm{R}$ and $\mathrm{S}$ strains.

midgut of the R strain. In contrast, the expression levels of the acsp30 remained unaltered upon feeding uninfected or parasite-infected blood in the S strain (data not shown).

\section{Structural analysis of acsp30 upstream regulatory sequences from $R$ and $S$ strains}

The cDNA corresponding to the acsp30 was cloned from both $\mathrm{R}$ and $\mathrm{S}$ strains and sequenced. A comparison of the two sequences did not reveal any difference between the two strains. Since the cDNA sequence of acsp30 was identical in both the strains, we isolated the corresponding genomic DNA using PCR. The 887 bp gDNA fragment was slightly bigger than the cDNA indicating the presence of an intron. Sequence analysis revealed that the gene has a single intron of $71 \mathrm{bp}$ at its $5^{\prime}$ end and is a phase 0 intron. Since the location and the sequence of the intron were identical in both the strains, the upstream sequences of $\operatorname{acsp} 30$ were isolated and evaluated for promoter activity. 
Using a PCR-based directional genome walking protocol, regions upstream to ascp30 were cloned from $\mathrm{R}$ and $\mathrm{S}$ strains. Amplicons of $1.4 \mathrm{~kb}$ and $0.7 \mathrm{~kb}$ were obtained in the $\mathrm{R}$ and $\mathrm{S}$ strains respectively. Upstream sequences $(702$ bases) from the R and S strains were aligned by ClustalW using MacVector (Version 7.0) and EMBOSS-Align (Fig. $3)$. A high degree of sequence similarity (94.2\%) was observed up to $333 \mathrm{bp}$, upstream of the translational start site (ATG). Beyond this region there was a considerable divergence in the gene sequence. The region between -702 bp and -333 bp showed $54 \%$ similarity with $41.7 \%$ gaps and only $30 \%$ similarity was observed in the region between -702 bp and -500 bp with $65.8 \%$ gaps (Fig. 4 ).

In silico analysis of the upstream sequences of acsp30 revealed characteristics of RNA polymerase II-transcribed promoters. Using the computer-based promoter prediction tool, two putative transcription start sites were predicted to be located at -31 bp (score cutoff of 1.0) and -40 bp (score cutoff of 0.8 ) upstream of ATG in R and S strains. The region surrounding this transcription start point corresponded to the arthropod initiator sequence, which is found in the interval -10 to +10 of $25 \%$ of arthropod RNA polymerase II-transcribed promoters as described by Cherbas and Cherbas (1993) [27]. This reinforced the prediction of this site as the true transcription start site. In both strains, two TATA motifs were found at position -53 and -189 from the ATG and two arthropod transcription initiator motifs TCAGT [27] were present at position -12 and -152 . Using the consensus DCAKTY [27], putative capsite was found at position -31 and -152 respectively in the R and $\mathrm{S}$ strains. These sites constituted the putative core promoter elements (Fig. 5 and Fig. 6).
Regulatory sequence motifs in both the strains were searched from within the insect family using the MatInspector Program [28] and 29 consensus matches were detected in the S strain and 19 in the R strain, all of which were based on factors present in Drosophila. In the S strain, the 29 different matrices were well distributed along the entire 702 bp upstream region but in the $\mathrm{R}$ strain such motifs were absent in the region between -304 bp and $702 \mathrm{bp}$ and the 19 matrices were restricted to the -1 to $303 \mathrm{bp}$ region. A comparative analysis of putative binding sites is depicted in Table 1.

The upstream regulatory sequences of the $\mathrm{R}$ and $\mathrm{S}$ strains were also scanned for putative vertebrate regulatory elements. Several interesting motifs were found in these regions. Computer-assisted analysis revealed motifs which were homologous to the response elements for transcription factors described in the promoters of other insect immune-responsive genes and mammalian acutephase protein genes and are listed in Table 2[29]. Of the several motifs listed, an interesting observation relates to the two potential regulatory motifs for the ecdysone responsive element (ECRE) (consensus RGKKSNNNGNNYK) [30]. In the R strain, EcRE are located at positions 199 to -211 bp (GGTTGAATGCGTT) and -5 to $-17 \mathrm{bp}$ (AGTGGTCAGTACT) on the plus strand while only one such motif was located in the $S$ strain at a position identical to the R strain (-199 to -211 bp). At present, it is difficult to assess the consequences of these differences on the refractory phenotype, a detailed mutational and deletion analysis is necessary to study the significance of these observations.

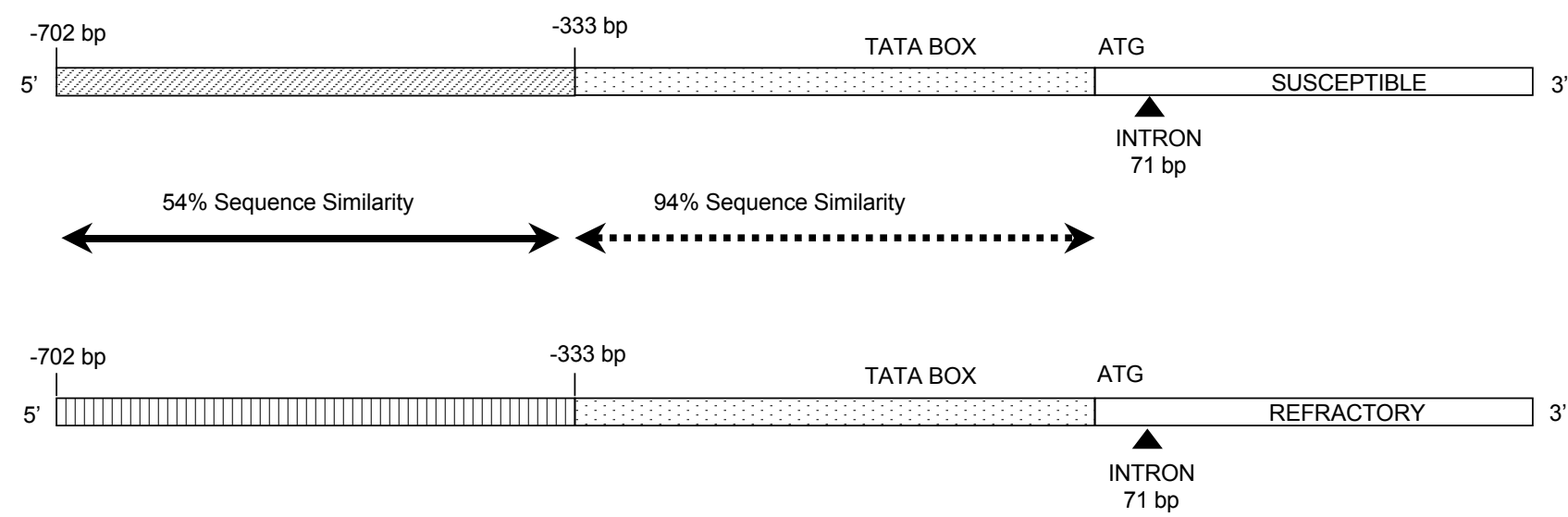

Figure 4

Comparative schematic diagram depicting the features of the upstream regions. Dotted line delineates the region displaying equal promoter activity and solid line delineates the region showing higher promoter activity in the $R$ strain than in the $S$. 
-702 TCСTGATCAАCTATATGGGTTCCTTGTAGCAGATCAACTTTACCAAGCAАTTGTGAT

Paired

-645 ACGAAAGTAAGAATGTA TATTAGCAAATTGATTCTTTCAATAGTATGATTACAATTT ICRE

Suppressor of hairless

Paired

-588 AAAAT TATCATTGAGCATCTGCATGCTGTGCGAAAGCTAGTAAATTTTTAATAAATG GATA2

Heat shock factor

-531 ATGAGATA IGAAAATAAATCGGCGCATTGTTTTGATCTGTTTTGTTTTGAGACTCC GATA2 ICRE GATA3

PAX6 P3

-474 AACCAAACATTTTGGCACACTACCCCAAAACCCAGCAATAATGCGGCTACCGTAAAA HNF 5

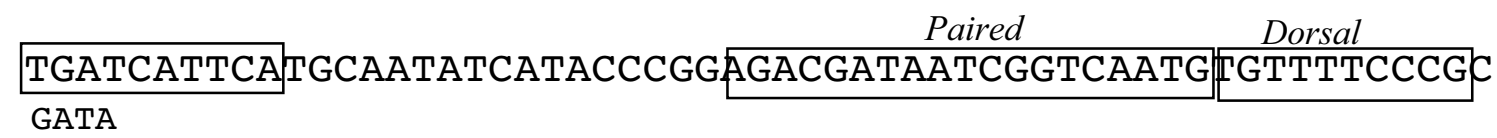

CGTACAATCTTTCAGAATAACGTCC GA $\overrightarrow{\text { TAAGAACGACCTGGCCGTCATtAqGAAATA }}$ GATA

NF-IL6

Hunchback РАХ6 P3

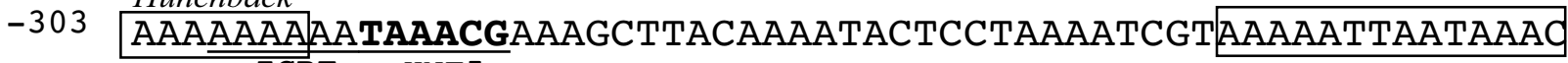
ICRE HNF5

EcRE

-246 TTATACACTGCAAGTGCCTGGTCATGTAACAGCTGGTTGAATGCGTTGGTTTAATAT

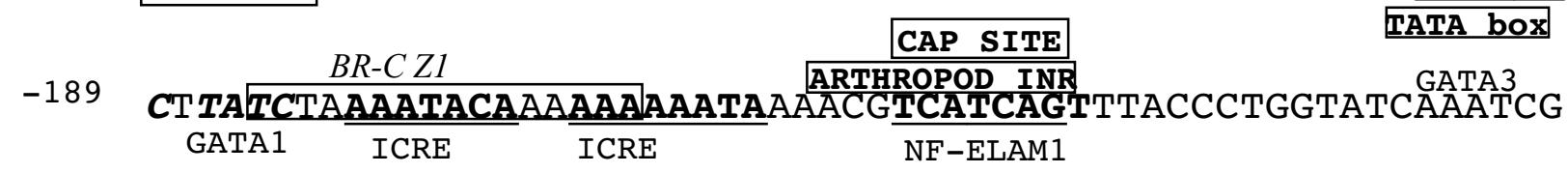

-132 АTATCACAAAАTTTTCTTACGAAGCGTATTTCAgCAATCTATCAAGTGTGTGACGCA NF-IL6

-75 CGGTGTGTATATATATATATAAGAGAAGACCCGGTAGCACGATAAGTCAATTGTCCG TATA box

-18 TAGAGGTCAGTACTAATC $\stackrel{\stackrel{+}{+1}}{\mathbf{T}}$ ARTHROPOD INR

\section{Figure 5}

Organization of the upstream regulatory sequences of acsp30 in the susceptible strain mosquitoes. Two TATA like boxes are present with corresponding arthropod initiator (Inr) sequences and capsites, which constitute the core promoter. Vertebrate upstream immune response elements are represented in bold fonts and underlined; hepatic nuclear factor 5 (HNF-5), nuclear factor interleukin 6 (NF-IL6), interferon consensus response elements (ICRE) and nuclear factor endothelial adhesion molecules (NF-ELAM I), ecdysteroid response elements (EcRE) and the different GATA factors are in bold face and italicized. Consensus sequences for all these motifs are described in the text. Insect regulatory sequence motifs are boxed. The numbers to the left denote the positions of the first nucleotide in each line, relative to ATG at +1 . The open block arrow indicates the predicted transcriptional start site (TSS). 


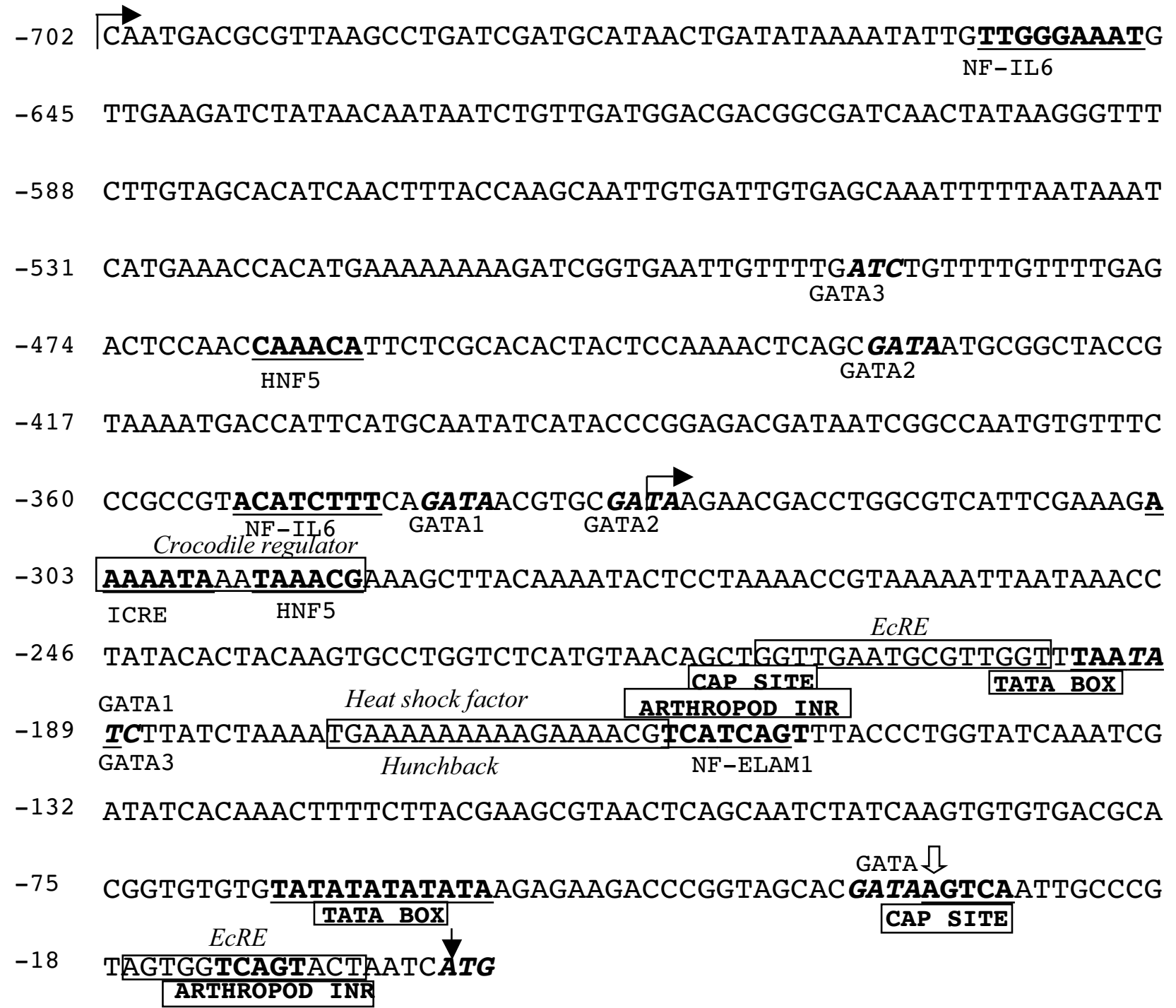

\section{Figure 6}

Organization of the upstream regulatory sequences of acsp30 in the refractory strain mosquitoes. Two TATA like boxes are present with corresponding arthropod initiator (Inr) sequences and capsites, which constitute the core promoter. Vertebrate upstream immune response elements are represented in bold fonts and underlined; hepatic nuclear factor 5 (HNF-5), nuclear factor interleukin 6 (NF-IL6), interferon consensus response elements (ICRE) and nuclear factor endothelial adhesion molecules (NF-ELAM I), ecdysteroid response elements (EcRE) and the different GATA factors are in bold face and italicized. Consensus sequences for all these motifs are described in the text. Insect regulatory sequence motifs are boxed. The numbers to the left denote the positions of the first nucleotide in each line, relative to ATG at +1 . The open block arrow indicates the predicted transcriptional start site (TSS).

\section{Functional analysis of the acsp30 promoter in $R$ and $S$ strains}

Based on the homology of upstream sequences of acsp30 between $\mathrm{R}$ and $\mathrm{S}$ strains, two regions were selected for evaluating the promoter activity. The region spanning -1 to -333 bp showed $94 \%$ similarity and the other region spanning -1 to -702 bp showed $72 \%$ similarity. These regions from the $\mathrm{R}$ and $\mathrm{S}$ strains were cloned in promoterless pGL3-Basic vector containing firefly luciferase reporter gene and transfected into Drosophila S2 cells. The luciferase activity from all the four constructs was higher than that of the vector (pGL3-Basic) and control (pGL3Control) suggesting that the upstream gene sequences from $\mathrm{R}$ and $\mathrm{S}$ strains contain regulatory elements for their 
Table I: Transcription binding sequences from the insect family in upstream regions.

\begin{tabular}{lll}
\hline Family/matrix & Refractory strain Position (from-to) & Susceptible strain Position (from-to) \\
\hline Drosophila paired homeodomain & Absent & $75-95$ \\
Suppresor of Hairless & Absent & $139-151$ \\
Drosophila paired homeodomain & Absent & $159-179$ \\
Heat shock factor (Drosophila) & Absent & $175-195$ \\
Drosophila PAX6 P3 homeodomain binding site & Absent & $275-295$ \\
Drosophila paired homeodomain & Absent & $315-335$ \\
Dorsal, protein for dorso-ventral axis formation (c-rel) & Absent & $33|-34|$ \\
Hunchback, early maternal and zygotic zinc finger gene & Absent & $394-406$ \\
Crocodile regulator of head developement & $404-416$ & Absent \\
Heat shock factor (Drosophila) & $529-549$ & Absent \\
Hunchback, early maternal and zygotic zinc finger gene & $529-54 I$ & Absent \\
\hline
\end{tabular}

respective promoters (Fig. 7). The two constructs pGL3Ref333 and pGL3-Sus333 yielded similar levels of luciferase activity, while pGL3-Ref702 from the $\mathrm{R}$ strain showed 1.5 -fold increase in luciferase activity compared to the $S$ strain.

\section{Differential binding of nuclear proteins to acsp30 upstream sequences from $R$ and $S$ strains}

The increase in promoter activity in the R strain was attributed to sequence differences within the 400 bp region spanning -333 to -702 bp upstream of the start codon. To determine the effect of such differences on the binding of nuclear proteins, electrophoretic mobility shift assays (EMSAs) were performed. EMSA experiments with three different probes (400 bp, $188 \mathrm{bp}$ and $100 \mathrm{bp}$ ) allowed us to determine the minimum upstream region that shows difference in binding of nuclear proteins and thus might be responsible for differential expression of acsp30 in the two strains. EMSAs using nuclear extracts from the R strain and R400/S400 probe revealed two complexes, a sharp slow migrating band, complex A and a faster moving diffused band, complex B (Fig. 8A, lane3). Both the com- plexes were observed when R188 probe was incubated with nuclear extract from R strain (Fig. 9, lane 3). Interestingly, formation of complex B was nearly abolished on R100 probe but complex A formation remained unaffected (Fig. 10). These results clearly indicated that the 88 bases (-602 to $-514 \mathrm{bp}$ ) missing in R100 probe were critical for the assembly of transcription factors forming complex B but the $100 \mathrm{bp}$ upstream region (-702 to $-602 \mathrm{bp}$ ) was important and sufficient for binding of nuclear proteins forming complex A.

In general, the binding of nuclear proteins to probes derived from the $S$ strain was less compared to that from the R strain, which further emphasized the importance of differences in upstream regions of acsp30 from both the strains. The greater intensity of the bands with the $\mathrm{R}$ probes showed that the formation of both the complexes was more on $\mathrm{R}$ probe than $\mathrm{S}$; an approximately $25 \%$ increase in DNA binding activity of both the complexes was observed on R400 as compared to S400 probe. Importantly, when S188 was used as a probe, there was an approximately $50 \%$ reduction in the formation of com-

Table 2: Putative transcription binding sequences in upstream region of refractory and susceptible strains of $A$. culicifacies that are homologous to insect immune responsive genes and vertebrate family.

\begin{tabular}{|c|c|c|}
\hline Putative binding sequences & Refractory strain Position (from-to) & Susceptible strain Position (from-to) \\
\hline Arthropod initiator & $\begin{array}{l}547-551 \\
691-695\end{array}$ & $\begin{array}{l}547-551 \\
691-695\end{array}$ \\
\hline Hepatic nuclear factor 5 & $\begin{array}{l}237-242 \\
408-413\end{array}$ & $\begin{array}{l}237-242 \\
408-413\end{array}$ \\
\hline GATA Factors (I, 2 and 3$)$ & $\begin{array}{l}664-676,356-368,512-524,266-278,366- \\
378,206-218,507-519\end{array}$ & $\begin{array}{l}36-376,664-676,5 I I-523,1 I 5-127,172-184, \\
20 I-213,506-518\end{array}$ \\
\hline $\begin{array}{l}\text { Interferon Consensus Response Elements } \\
\text { (Icre) }\end{array}$ & $399-405$ & $\begin{array}{l}60-66,68-74,|8|-\mid 87,404-410,522-528, \\
532-538\end{array}$ \\
\hline Mammalian Type I-Interleukin Response & $48-56$ & $399-398$ \\
\hline Element (Nf-II6) & $350-358$ & $600-608$ \\
\hline $\begin{array}{l}\text { Nuclear Factor Endothelial Leucocyte } \\
\text { Adhesion Molecule (NF-ELAMI) }\end{array}$ & $544-550$ & $544-550$ \\
\hline Nf-Kappa B & Absent & Absent \\
\hline
\end{tabular}




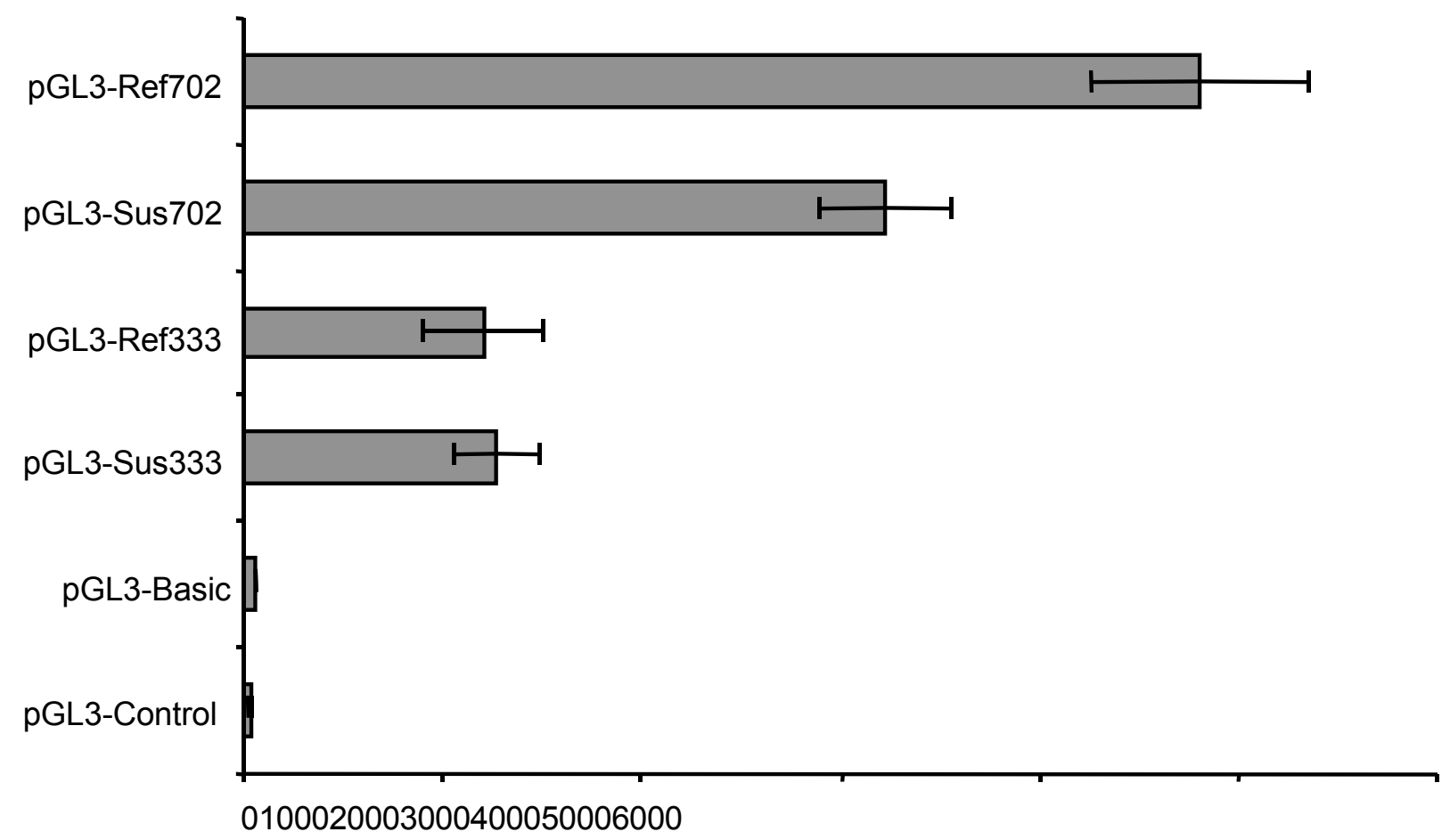

Relative Light Units

\section{Figure 7}

Assessment of promoter activity of acsp30 from $\mathbf{R}$ and $\mathbf{S}$ strains of $A$. culicifacies using luciferase-based reporter assay in Drosophila S2 cell line. Constructs, pGL3-Ref (702 bp and 333 bp) and pGL3-Sus (702 bp and 333 bp) were transfected in the Drosophila Schneider (S2) cell line using lipofectin and the luciferase activity measured. Promoterless vector pGL3Basic and pGL3-Control plasmid with the SV40 promoter served as controls. Reported activities were based on three independent transfections and the data was recorded in relative light units (RLU).

plex B than on R188. This could be a consequence of an increase in sequence divergence $(70 \%)$ in this region between the two strains.

Specificity of interaction of nuclear proteins with various probes from $\mathrm{R}$ and $\mathrm{S}$ strains was evaluated by competition assays in the presence of corresponding specific cold probe. The binding of nuclear factors to $400 \mathrm{bp}$ upstream sequence from $\mathrm{R}$ strain (R400) was highly specific as the formation of complexes A and B was reduced to $25 \%$ in presence of 100-fold molar excess of unlabeled $400 \mathrm{bp}$ cold probe in the EMSA binding reaction mixture (Fig. 8). Competition experiments with sequentially shorter fragments from $\mathrm{R}$ and $\mathrm{S}$ strains also generated similar results showing the specificity of binding of nuclear factors to all the probes (data not shown).

We also performed EMSA experiments using nuclear extract from both the strains to evaluate the presence of additional transcription factors in the $\mathrm{R}$ strain that could be absent in the S strain. Noticeably, the binding pattern of nuclear proteins from S strain to S188 and R188 probes (Fig. 9) were different from that of nuclear proteins from $\mathrm{R}$ strain. A similar result was obtained with $\mathrm{S} 100$ and R100 probes (Fig. 10). When a nuclear extract from the S strain was used with R100 probe, the faster migrating band, complex B did not form. This is indicative of either a lack of the transcription binding factors in the $S$ strain that form complex B or their low concentrations that prevent detection. The association of putative binding factors was quantified by converting intensity of signals to numerical values by using the Image Analysis Software, ImageQuant TL (Amersham Biosciences) and the results were presented as bars in figures, 5, 6 and 7 .

\section{Discussion}

Naturally occurring, malaria-resistant strains, like the refractory strain of Anopheles culicifacies are very rare in the 


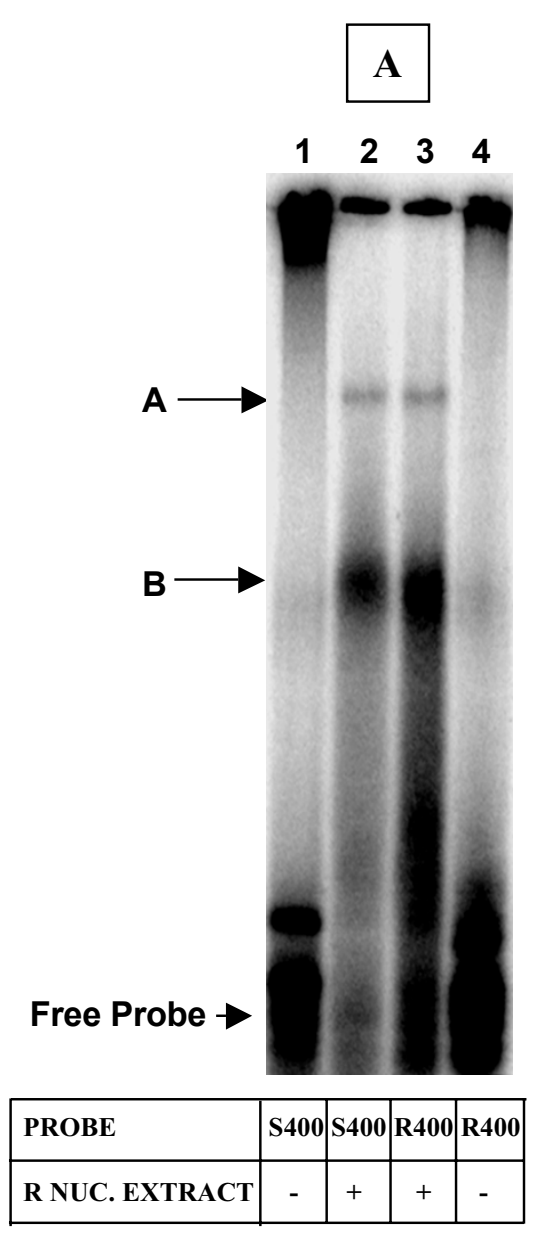

Complex AComplex B

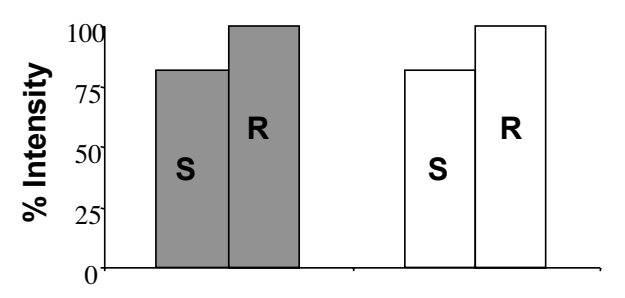

B

$\begin{array}{lllll}1 & 2 & 34 & 5\end{array}$

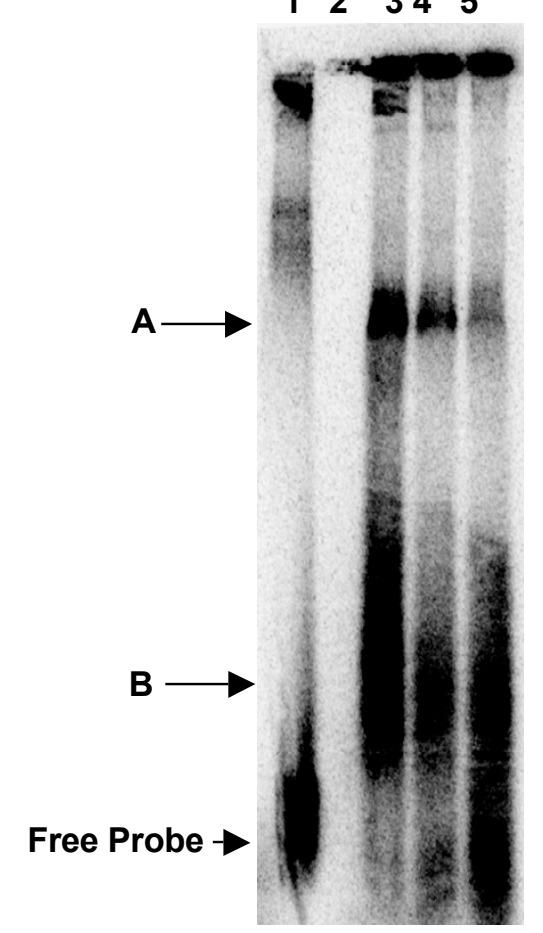

\begin{tabular}{|l|cccc|}
\hline PROBE-R400 & + & + & + & + \\
\hline R. NUC. EXTRACT & - & + & + & + \\
\hline COLD COMPETITOR & - & - & $50 X$ & 100X \\
\hline
\end{tabular}

Complex A Complex B

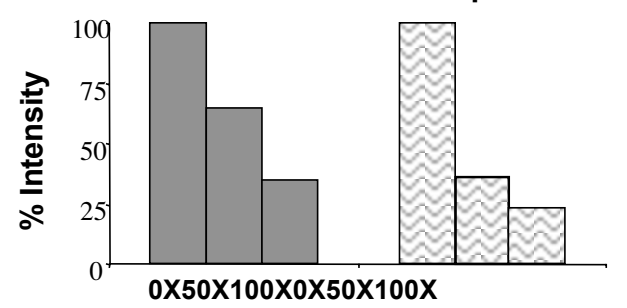

\section{Figure 8}

EMSAs with $A$. culicifacies refractory $(R)$ strain nuclear extract and $400 \mathrm{bp}$ probes from upstream sequences of acsp30 of S and R strains. (A) EMSA showing the binding pattern of nuclear proteins extracted from body tissue of five-day old adult female R strain mosquitoes when incubated with $S 400$ (lane 2) and R400 (lane 3) probes at $37^{\circ} \mathrm{C}$ for 25 minutes. Free probes were run in lane I (S400) and lane 4 (R400). (B) The radiolabelled probe R400 was incubated with refractory nuclear extracts without competitor (lane 3 ) and in the presence of unlabeled probe at 50-fold (lane 4) and 100-fold (lane 5) molar excess. Arrows indicate the migration of complex A (slow migrating) and B (fast migrating). The intensities of the signals were quantified with respect to Phospholmager signals by the Image Analysis Software, ImageQuant TL (Amersham Biosciences) and represented as the percentage ratio of S400 to R400 signal intensities (Fig. 8A) and as percentage ratio to the uncompeted (R400) signal intensities (Fig. 8B). 


\section{A}

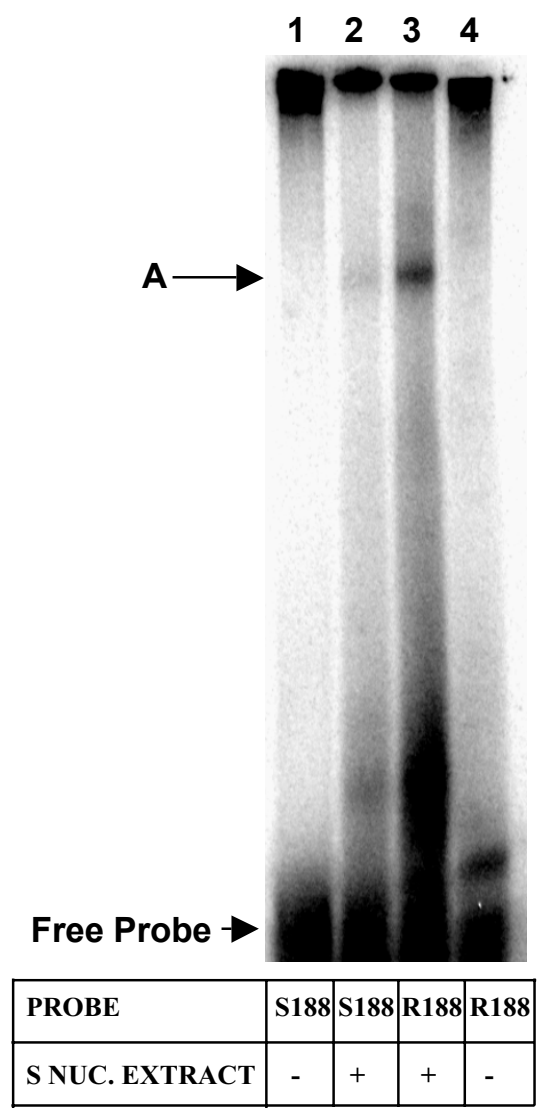

Complex A

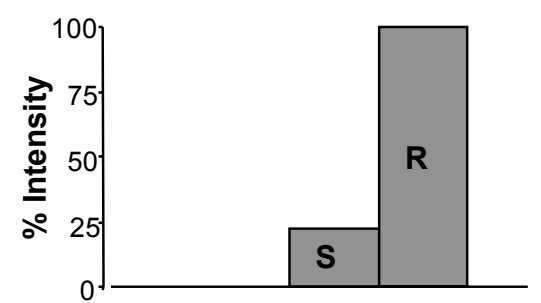

\section{B}

$\begin{array}{llll}1 & 2 & 3 & 4\end{array}$

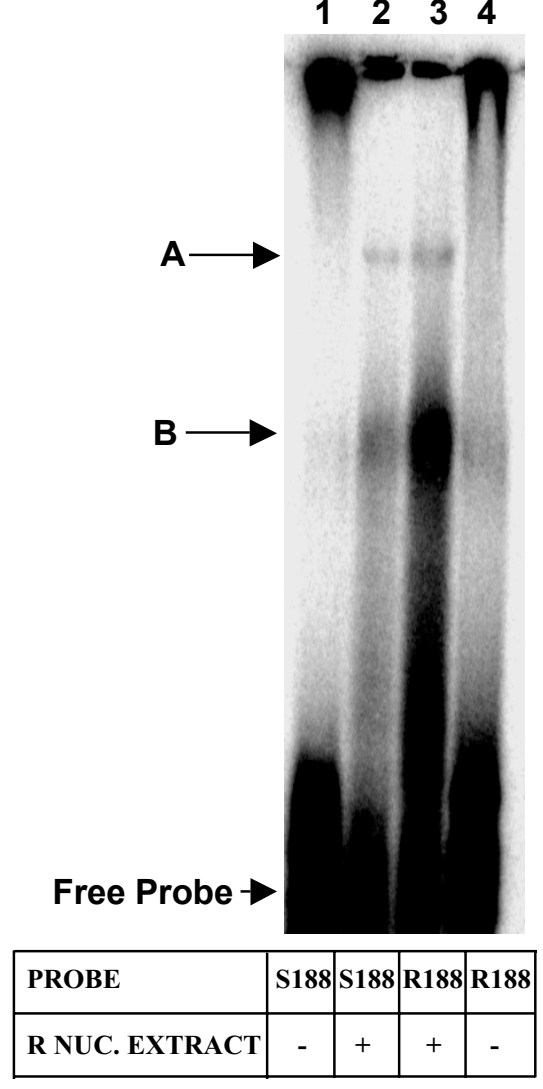

Complex AComplex B

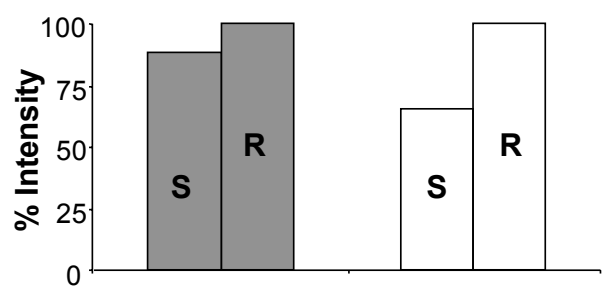

\section{Figure 9}

EMSAs using nuclear extracts and 188 bp probes from acsp30 upstream sequences. Nuclear proteins were extracted from body tissue of 5-day old adult female of S strain (A) and R strain (B) and incubated with SI88 (lane 2) and RI88 (lane 3) probes. Free probes were run in lane I (SI88) and lane 4 (RI88). Arrows indicate the migration of complex A (slow migrating) and complex $B$ (fast migrating). The intensities of the signals were quantified with respect to Phospholmager signals by the Image Analysis Software, ImageQuant TL (Amersham Biosciences) and represented as the percentage ratio of SI 88 to RI88 signal intensities.

field. Low occurrence of such resistant phenotype is attributed to the selection pressures on the immune system that are responsible for strain survival and reproductive success. Genetic analyses of this strain have revealed that the genes for refractoriness in A. culicifacies are dominant and autosomal [8]. Here, we present analyses of one of the putative biochemical factors of natural population of $A$. culicifacies mosquito that displays refractoriness to Plasmo- 


\section{A}

\section{$\begin{array}{lll}12 & 3 & 4\end{array}$}

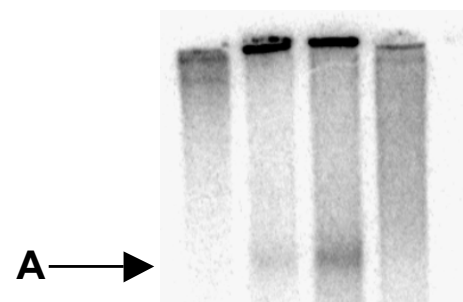

Free Probe

\begin{tabular}{|l|l|l|l|l|}
\hline PROBE & S100 & S100 & R100 & R100 \\
\hline S NUC. EXTRACT & - & + & + & - \\
\hline
\end{tabular}

Complex A

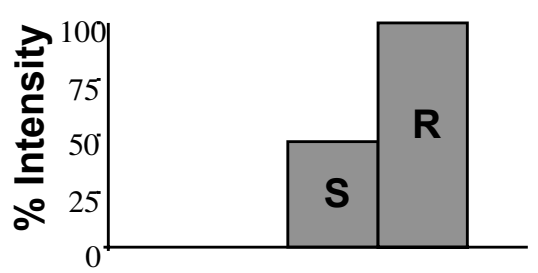

B

$\begin{array}{llll}1 & 2 & 3 & 4\end{array}$

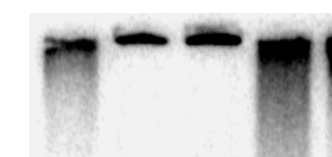

A

Free Probe -

\begin{tabular}{|l|c|c|c|c|}
\hline PROBE & S100 & S100 & R100 & R100 \\
\hline R NUC. EXTRACT & - & + & + & - \\
\hline
\end{tabular}

\section{Complex A}

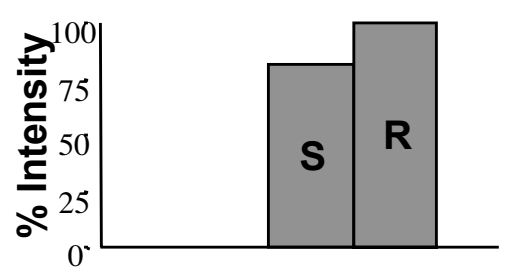

Figure 10

EMSAs using nuclear extracts and 100 bp probes from acsp30 upstream sequences. Nuclear proteins were extracted from body tissue of 5-day old adult female of $S$ strain $(A)$ and R strain (B) and were incubated with $S I 00$ (lane 2 ) and RI00 (lane 3) probes. Free probes were run in lane I (SI00) and lane 4 (RI00). Arrows indicate the migration of complex $A$ (slow migrating) and complex B (fast migrating). The intensities of the signals were quantified with respect to Phospholmager signals by the Image Analysis Software, ImageQuant TL (Amersham Biosciences) and represented as the percentage ratio of SI00 to RI00 signal intensities. 
dium by melanotic encapsulation of ookinetes. The phenoloxidase cascade that is responsible for the melanization event recruits serine protease to activate prophenoloxidase, thereby leading to the formation of phenoloxidase, tyrosine and quinones [12]. Serine proteases are reported to play a determinant role in triggering melanotic encapsulation of parasites in many malariarefractory mosquito strains $[5,18,31]$.

We focused our analysis on one of the early determinants of encapsulation phenotype, serine proteases. A parasiteinducible serine protease-encoding gene (acsp30) was isolated from the body tissue of $A$. culicifacies. The deduced protein, ACSP 30 , shared $30 \%$ or less sequence similarity with serine proteases from other insects such as Drosophila, Chrysomia and Culex. These serine proteases are reported to function mainly in dissolution of fibrin clots or in embryonic development. Notably, ACSP30 did not possess a CLIP domain and shared a very low sequence homology $(<15 \%)$ with Clip domain serine proteases that are implicated in melanization of malaria parasites $[18,20,32]$. It may represent a divergent class of hitherto undescribed serine protease that plays a role in melanotic encapsulation of the parasite.

Importantly, transcriptional analysis of acsp30 revealed that it could play an important role in the mosquito immunity. Real time analysis demonstrated that the levels of acsp30 were 40-fold higher in the refractory strain as compared to the susceptible strain even in the absence of any challenge. The strain seems to have evolved the capacity to constitutively express high levels of serine protease, which could serve as a molecular marker for differentiating $\mathrm{R}$ and $\mathrm{S}$ strains. The high transcript levels of acsp30 in the $\mathrm{R}$ strain prompted us to further investigate its role inresponse to parasite invasion. In this regard, timedependent experiments that involved feeding $\mathrm{R}$ and $\mathrm{S}$ mosquitoes uninfected and Plasmodium-infected blood were carried out. Expression levels of acsp30 were significantly increased upon un-infected blood feeding (68-fold over the naïve unfed control) in the R strain but no such induction was observed in the $S$ strain, thereby making it a strain-specific response. Upon feeding parasite-infected blood, the transcription of acs $p 30$ was further upregulated (4.4-fold over the uninfected blood-fed control) suggesting that endogenous levels of the transcript were not sufficient to combat the invading parasite and an added induction was required to block parasite development. Noticeably, upregulation of acsp30 transcript levels 24 hours after parasite-infected blood feeding coincided well with the appearance of melanotically encapsulated ookinetes in midgut of the $\mathrm{R}$ strain of A. culicifacies.

Both, time and high level of induction of acsp30 are suggestive of its role in PPO-mediated melanization cascade triggered in response to Plasmodium. Importantly, the transcript levels of acsp30 were unaffected when the nonmelanizing S strain was fed on Plasmodium-infected blood (data not shown). This result further strengthened the possibility of involvement of acsp30 in contributing towards the refractory phenotype to the mosquito along with other genes. Interestingly, when the $\mathrm{R}$ strain of $A$. culicifacies was challenged with a gram-positive bacterium, Micrococcus luteus (data not shown), the expression levels of acsp30 were not affected, indicating that the induction of acsp30 was a Plasmodium-specific response.

Recently, gene silencing of Clip domain serine proteases by RNAi have revealed their involvement in killing of parasite and melanization of sephadex beads [18-20]. The loss of refractory phenotype of the $\mathrm{R}$ strain mosquitoes upon injection of dsRNA corresponding to acsp30 could illuminate the specific role of ACSP30 in melanization. The steps that lead from upregulation of acsp30 to encapsulation in the refractory strain are not clear at present. It is possible that upregulation may be a postmelanotic event facilitating clearance of parasite [20].

To understand the basis of differential expression of acsp30 in the R and S strains, structural analysis of the gene was carried out. Genomic DNA and CDNA sequences of acsp30 from both strains were found to be identical, including the presence of a $71 \mathrm{bp}$ intron. Therefore, we concluded that the coding and the non-coding regions of the gene were not responsible for the observed differential expression in the two strains. The upstream sequences of the gene from both the strains were cloned, scanned and evaluated for promoter activity using a promoterless, luciferase reporter vector. The promoter activity of the $333 \mathrm{bp}$ regions was nearly identical whereas 702 bp region from the R strain yielded 1.5-fold higher luciferase activity than the $S$ strain. This observation suggested that the sequence between -702 bp and -333 bp was responsible for the differences in promoter activity in $\mathrm{R}$ and $\mathrm{S}$ strains. Sequence alignment of the $400 \mathrm{bp}$ region (-333 to $-702 \mathrm{bp}$ ) from both strains revealed $54 \%$ similarity compared to $94 \%$ similarity in the 333 bp region ( -1 to $-333 \mathrm{bp})$ thereby providing support to above observation.

An in silico analysis of acsp30 upstream sequences was carried out using the MatInspector program. It is premature to identify amongst the listed transcription factors any correlative specifics for encapsulation, nevertheless, one particular transcription factor that deserves special mention is Dorsal. It is mainly engaged in transcriptional activation of genes involved in dorsoventral patterning [33]. The mosquito orthologue of dorsal is Gambifl (REL1), which has been shown to translocate to the nucleus following bacterial but not Plasmodium infection [34]. Inter- 
estingly, this site was present in the upstream sequences of acsp30 from susceptible but not from the refractory strain.

Several signature sequence motifs implicated in promoters of immune-responsive genes from $A$. gambiae and Aedes aesypti [29,35-37] were also present within the upstream sequences of $a c s p 30$. Notably, the consensus for NF-kB, which is reported in Aedes [35] and A. gambiae [29] defensin genes is absent in acsp30, suggesting that defensin pathway-associated serine protease is different from the phenoloxidase-associated serine protease. Interestingly, the A. gambiae PPO1 gene that codes for melanin-synthesizing enzyme prophenoloxidase is the first mosquito immune gene reported to contain two ecdysteroid response elements (EcRE's) in its promoter sequence [38]. Using reported consensus sequences, two putative EcREs were located in acsp30 upstream sequences from the R strain and one in the S strain [30]. The significance of these differences and their eventual effect on refractoriness in A. culicifacies remains to be ascertained. A closer look at the nucleotide composition of the upstream sequences in the two strains revealed a very high $\mathrm{G}+\mathrm{C}$ content in the $\mathrm{R}$ sequences and high $\mathrm{A}+\mathrm{T}$ in the $\mathrm{S}$ sequences which probably could influence the affinities or the strength of the DNA-protein interactions.

EMSA experiments were carried out to further characterize the upstream sequences of acsp30 from both strains. Together with $400 \mathrm{bp}$, which showed higher promoter activity in R strain, two more regions within (100 and 188 bp) were chosen for EMSA experiments. Nuclear proteins from the $\mathrm{R}$ strain mosquitoes interacted with R400 probe to form two complexes, larger complex $\mathrm{A}$ and smaller complex B. The complex A appeared as a discrete band whereas complex B was observed as a diffused band in the agarose gel with greater intensity than complex A. This could be due to greater affinity of complex $\mathrm{B}$ for the probe than complex A or due to its higher abundance. The sequence differences between R400 and S400 led to a $25 \%$ reduction in formation of both the complexes on S400, showing an overall decrease in number of transcription factors binding the upstream sequences of acsp30 in the $\mathrm{S}$ strain as compared to the $\mathrm{R}$ strain. Competition binding experiments demonstrated that binding of nuclear proteins for complex formation was highly specific. By using two deletion mutants of the 400 bp region, we were able to narrow down the regions that were important for the assembly of both complexes.

The formation of complex A was seen even in the smallest, $100 \mathrm{bp}$ region used for EMSA experiments but complex B was completely abolished in this region and required an additional 88 bases. Importantly, S strain nuclear extracts lacked or had very low levels of transcription binding factors (TBF) forming complex B. Complex A formation remained unaffected on using nuclear extract from the $S$ strain, indicating that the nuclear proteins forming complex A were present in both the strains but, the sequence difference between $\mathrm{R}$ and $\mathrm{S}$ upstream sequences was critical for its formation. On the other hand, formation of complex $\mathrm{B}$ was sensitive to both the sequence and the presence of transcription binding factors in the nuclear extract. Identification and characterization of these TBFs would be necessary to further evaluate the differences in the two strains. The 2-D analysis of nuclear proteins from $\mathrm{S}$ and $\mathrm{R}$ strains will probably reveal the identity of the TBFs responsible for high level expression of acsp30 transcript and other genes contributing to the refractory phenotype.

\section{Conclusion}

In conclusion, this is the first report of an immuneresponsive serine protease from a field-collected refractory strain of Anopheles culicifacies, the main vector of malaria in India. Transcript abundance of acsp30 in naïve adult refractory mosquitoes, even in the absence of any immune challenge, suggests possibility of its contribution towards refractory phenotype to A. culicifacies population. Through a series of EMSA experiments and in silico analysis, we have tentatively identified potential regulatory binding motifs that could be responsible for differential expression of acsp30 in R and S strains of A. culicifacies. Further analysis of factors responsible for conferring refractory phenotype to mosquito against malaria parasite may pave way towards identification of critical intervening steps to abort parasite development in mosquito.

\section{Methods \\ Mosquito rearing}

Cyclic colonies of S and R strains of malaria vector, Anopheles culicifacies, were reared in an insectary at National Institute of Malaria Research, Delhi maintained at a temperature of $28 \pm 1{ }^{\circ} \mathrm{C}$ and $75 \pm 5 \%$ relative humidity and fitted with simulated dusk and dawn machine with a photoperiod of 14 hour day and 10 hour night as described by Adak et al. (1999) [39].

Mosquitoes were fed upon 1\% glucose soaked pads and raisins. Female mosquitoes were offered rabbit blood for ovarian development. Following hatching, larvae were reared in enamel trays containing de-chlorinated water and fed on powdered dog biscuits and brewer's yeast tablets in 3:2 ratios.

\section{Cloning of ascp30 of A. culicifacies}

Total RNA was isolated from the body tissue of refractory population of A. culicifacies by the TRIZOL Reagent method according to the manufacturer's instructions (Invitrogen, USA). To avoid contamination of the digestive proteases, the gut was removed from the mosquitoes asep- 
tically. Up to $5 \mu$ g total RNA was used for cDNA synthesis using reverse transcriptase enzyme (Superscript II) and adapter primer (AP, 5' GGC CAC GCG TCG ACT AGT ACT TTT TTT TTT TTT TTT $\left.3^{\prime}\right)$.

Degenerate primers, SerPr1 (5'-TGG GTC/G C/GTG ACC/ G GCC/G GCG/A/T/C CAC/T-3') and SerPr2 (5'-ACG AGC/G CGA/G CCA/G CCC/G GAA/G TCG/A/T/C-3') were designed based on the sequence of reported serine protease in A. gambiae [21]. Using these degenerate primers and cDNA as template, a $450 \mathrm{bp}$ fragment was amplified. The fragment was cloned into pGEM-Teasy vector (Promega Corporation, USA) and sequenced by Microsynth (Switzerland). On the basis of sequence of cloned insert, a set of gene-specific primers, DSerPr1 (5'CGT GCA CTT GAA CAT TAT CTC-3') and DSerPr2 (5'CTG GTT GGC AGC GTC ACG A-3') were synthesized, to obtain 5' and 3' ends of the gene respectively, by RACE (rapid amplification of cDNA ends) according to the protocol described in the 5' and 3' RACE kit manuals (Invitrogen). Gene-specific end primers, 10FMOS (5'-ATG AAA CTG TTC ATC GTC GT-3') and 11R (5'-TTC AGT ACT TGA TGC CAG ATT-3') were designed and synthesized on the basis of sequences of 5' and 3' RACE fragments to obtain the full-length serine protease gene. Using these end primers and cDNA as template, a complete 816 bp gene (ascp30) was isolated, cloned in pGEMT-easy vector and sequenced. All the primers were synthesized from Microsynth.

\section{Dissections and RNA extractions}

The mosquitoes were dissected on clean new slide in a drop of ice-cold sterile DEPC-treated water and body tissue (abdomen and thorax) was collected by removing midgut, heads, legs and wings. Body tissue from ten mosquitoes was pooled in $500 \mu \mathrm{l}$ of TRIZOL. Mosquito larvae of the different instars were also collected and stored in TRIZOL at $-70^{\circ} \mathrm{C}$.

The tissues were ground to homogeneity with a batterydriven hand-held homogenizer using DEPC-treated sterile grinder tips and processed for RNA extraction by TRIZOL Reagent method. Contaminating DNA was removed by treatment with RQ1 DNase (Promega Corporation) according to manufacturer's instructions. The total RNA isolated was used as a template for semi-quantitative and real time RT-PCR analysis of acsp30 transcripts using $\beta$ actin as internal control.

\section{Blood feeding strategy}

Rodent malaria parasite, Plasmodium vinckei petteri 279BY, supplied by Prof. Irene Landau, Museum National d'Historie Naturelle, Paris [41], was maintained on rodentmosquito cycle at the National Institute of Malaria Research, Delhi [7]. Cryo-preserved Plasmodium vinckei petteri infected blood isolates $(500 \mu \mathrm{l})$ were mixed with an equal volume of incomplete RPMI culture media and were inoculated into healthy 4-5 weeks old Balb/c mice. Thin blood smears prepared from peripheral blood of infected mice were fixed in methanol and stained in JSB stain [42]. The slides were examined under oil immersion lens of compound microscope (Carl-Zeiss, Germany) for the presence of various stages of parasite. Ideal time for feeding was ascertained by periodically monitoring the parasitemia of infected mice and looking for the presence of at least $0.5 \%$ mature gametocytes. Four- to six-day old mosquitoes were starved by depriving them of raisin and glucose pads for nine hours. Approximately 100-200 starved mosquitoes were held in cages and gametocytepositive mouse was placed in the cage for 1 hour during daytime for feeding. Subsequently, the full-fed mosquitoes were separated from unfed and partially-fed mosquitoes in a separate cloth cage labeled accordingly and were maintained at $23-24^{\circ} \mathrm{C}$ temperature and $65-70 \%$ relative humidity in the insectary. Mosquitoes were dissected after $6,12,18$ and 24 hours of blood feeding and the tissues were stored in TRIZOL at $-70^{\circ} \mathrm{C}$ until analysis.

\section{Semi-quantitative RT-PCR analysis}

The relative transcript abundance of acsp30 in naïve mosquitoes ( $\mathrm{S}$ and $\mathrm{R}$ strains) was determined by semi-quantitative RT-PCR using a One-step RT-PCR kit (Qiagen $\mathrm{GmbH}$, Germany). The amount of RNA in both the tissues was normalized to the $\beta$-actin gene. The sequences of the primer pairs used for amplifying $\beta$-actin were $\beta$-actinFor (5'-CAG ATC ATG TTT GAG ACC TTC AAC-3') and $\beta$-actinRev (5'-GA/C/TC CAT CTC C/TTG CTC GAA A/GTC-3'). Using the normalized amount of RNA as template, ascp30 transcript was amplified by using gene-specific primers, forward 10F (5'-ATCAGTTACCAATCGATCTTGCC-3') and reverse 4R (5'-CGTACGTTCCCATGCACTG-3') to obtain a 500 bp amplicon. The amplification regimen was as follows: reverse transcription at $48^{\circ} \mathrm{C}$ for 30 minutes, inactivation at $95^{\circ} \mathrm{C}$ for 15 minutes and the PCR at $94^{\circ} \mathrm{C}$ for 30 seconds, $52^{\circ} \mathrm{C}$ for 30 seconds and $72^{\circ} \mathrm{C}$ for $30 \mathrm{sec}-$ onds for 33 cycles followed by a final extension of $72^{\circ} \mathrm{C}$ for 10 minutes. Before loading on 1\% agarose gel, the RTPCR products were treated with $1 \mu \mathrm{g}$ of RNase (Qiagen $\mathrm{GmbH})$ at $37^{\circ} \mathrm{C}$ for 10 minutes to eliminate template RNA. The gel was photographed with Polaroid 667 black and white print film.

\section{Real Time RT-PCR analysis}

Real time RT-PCR was performed using Quanti Tect SYBR Green RT-PCR kit (Qiagen GmbH) and iCycler TM system (Bio-Rad Laboratories, USA) to measure relative transcript levels of acsp30 in naïve $\mathrm{R}$ and $\mathrm{S}$ mosquitoes, in various developmental stages of $\mathrm{R}$ strain mosquito and after blood-feeding and parasite-feeding treatments. The Primer 3 web-based tool was used to design gene-specific 
primers, ensuring that the length of the PCR product was 300 bp. $\beta$-actin amplicon was obtained by using $\beta$-actinFor and $\beta$-actinRev primers and acsp30 amplicon was obtained by using AcSp30For (5'-GTC AGA CCG CTG GTG GTA AT-3') and AcSpRev (5'-CTC ACG GTT GAG GAA CGT CT-3') primers. Each $25 \mu$ reaction mixture contained $2 \mu \mathrm{l}$ of template RNA $(100-500 \mathrm{ng} / \mu \mathrm{l}), 2 \times$ QuantiTect SYBR Green RT-PCR Master Mix, $2 \mu \mathrm{l}$ of primers (5 pmoles/ $\mu \mathrm{l})$, RNase-free water and $0.25 \mu \mathrm{l}$ of QuantiTect RT enzyme Mix. Real-time cycler conditions included a preliminary reverse transcription at $48^{\circ} \mathrm{C}$ for 30 minutes, an initial activation step at $95^{\circ} \mathrm{C}$ for $15 \mathrm{~min}$ utes and 40 cycles of denaturation $\left(94^{\circ} \mathrm{C}\right)$, annealing $\left(52^{\circ} \mathrm{C}\right)$ and extension $\left(72^{\circ} \mathrm{C}\right)$ for 30 seconds each. The final step included gradual temperature increase from $50^{\circ} \mathrm{C}$ to $94^{\circ} \mathrm{C}$ at the rate of $1^{\circ} \mathrm{C} / 10$ seconds to enable melt-curve data collection. A non-template control (NTC) was run with every assay. The threshold cycles $\left(C_{T}\right)$ were recorded for acsp30 and $\beta$-actin amplicons during each experiment. Difference between the $\mathrm{C}_{\mathrm{T}}$ of $\beta$-actin and acsp30 or $\Delta \mathrm{C}_{\mathrm{T}}$ was determined and the relative abundance of acsp30 was calculated in different treatments using Comparative $\mathrm{C}_{\mathrm{T}}$ method using the formula $2^{-\Delta \Delta C T}$ [43].

\section{Isolation of genomic clone of acsp30}

Genomic DNA (gDNA) was isolated from the body tissue of $\mathrm{R}$ and $\mathrm{S}$ strains of mosquito by the method of Henry et al., 1990 [40]. Using gDNA as template and end primers, $10 F M O S$ and $11 \mathrm{R}$, the genomic clones of acsp30 from both strains were amplified by PCR. The obtained amplicons were cloned into pGEM-Teasy vector, sequenced by ABI PRISM and a comparative analysis of the nucleotide sequence from both strains was done using the ClustalW (MacVector Version 7.0).

\section{Isolation and cloning of upstream regulatory sequences} Three nested gene-specific reverse primers; R1 (Biotinylated) (5'-ACTACGAGGAGATTCACGCATGG-3'), R2 (5'-TTA TTCACACCTTGAGTTCAATTG-3') and R3 (5'GGCTAAAACGACGACGATGAACAG-3') were designed from the 5 ' end of serine protease cDNA sequence. Using $50 \mathrm{ng}$ of genomic DNA as template, PCR was performed in four different tubes with four forward walker primers (WP) [WP1, 5'-CTAATACGACTCACTATAGGGNNNNATGC-3'; WP2, 5'-CTAATACGACTCACTATAGGGNNNNGATC-3'; WP3, 5'CTAATACGACTCACTATAGGGNNNNTAGC-3'; WP4, 5'CTAATACGACTCACTATAGGGNNNNCTAG-3'] and the reverse biotinylated primer, $\mathrm{R} 1$ according to the directional walking method described by Mishra et al., 2002 [44]. Amplified biotinylated products were then immobilized on streptavidin-linked paramagnetic beads and nonbiotinylated DNA was washed off. Nested PCR was carried out using the above immobilized PCR product as template and gene-specific primer, R2 and T7-based forward walker primer (5'-CTAATACGACTCACTATAGGG-3'). Amplified PCR products were cloned into pGEM-Te vector and sequenced by Microsynth.

Forward primers containing the KpnI site were designed and synthesized. They were located at 702 bp and $333 \mathrm{bp}$ upstream to the translational start site (ATG) at +1 position. These included; RefDelFor702, (5'-GGTACCCAATGACGCGTTAAGCC-3'), RefDelFor333, (5'GGTACCGATAAGAACGACCTGG-3') for the refractory strain and SusDelFor702, (5'-GGTACCTCCTGATCAACTATAT-3'), SusDelFor333, (5'-GGTACCGATAAGAACGAC CTG G-3') for the susceptible strain. The reverse primers were designed from a region just upstream to the ATG and contained XhoI restriction site. These included, RefSPRev, (5'-CTCGAGGATTAG TACTGACCACTACGG$\left.3^{\prime}\right)$ for the R strain and SusSPRev, (5'-CTCGAGGATTAGTACTGACCTCTACGG-3') for the S strain. Using the DNA of the pGEM-Teasy clones as template and the above primers, a 32 cycle PCR was carried out with the following conditions; $94^{\circ} \mathrm{C}$ for 30 seconds, $52^{\circ} \mathrm{C}$ for 30 seconds and $72^{\circ} \mathrm{C}$ for 1 minute with a final extension at $72^{\circ} \mathrm{C}$ for 5 minutes. PCR products were again cloned into pGEMTeasy vector. Plasmids prepared from the positive colonies were double digested with KpnI and XhoI. The fallout was eluted from the gel using Qiagen gel extraction kit and ligated into promoter-less luciferase reporter vector pGL3 Basic (Promega Corporation), which was pre-digested with KpnI and XhoI enzymes. Resulting reporter plasmids were named pGL3-Ref702, pGL3-Sus702, pGL3-Ref333 and pGL3-Sus333.

\section{Computer-based sequence analysis}

The upstream sequences of acsp30 isolated from R and S strains were analyzed for potential transcription factor binding sites using the MatInspector program [28]; matrix library was set as Insect library, the core similarity at 0.75 and optimized matrix similarity was used for both the strains. The upstream sequences for the two strains were aligned using the ClustalW program (MacVector Version 7.0) and EMBOSS alignment programs. The core promoter elements and other vertebrate transcription binding factors were identified using reported consensus sequences as described in the results.

\section{Cell culture, transfections and luciferase assay}

Drosophila S2 cells were obtained from Invitrogen and were maintained at $27^{\circ} \mathrm{C}$ in Schneider's Drosophila medium (Invitrogen) with $10 \%$ heat inactivated fetal bovine serum in $25 \mathrm{~cm}^{2}$ cell culture flasks. One day before transfection, S2 cells were seeded at $0.75 \times 10^{6}$ cells $/ \mathrm{ml}$ in 24 -well tissue culture plates to achieve $80 \%$ confluency. DNA $(1.5 \mu \mathrm{g})$ was transfected using cellfectin (Invitrogen) according to the manufacturer's instructions. The transfection was carried out in serum-free medium for 8-12 
hours. The transfection medium was then replaced with serum-containing medium and the cells were harvested 48 hours after transfection in $50 \mu \mathrm{l}$ of $1 \times$ Cell Culture Lysis Reagent. Luciferase activity was then measured using the Luciferase assay system (Promega Corporation) and the luminescence was read on a Packard LumiCount manual luminometer. A pGL3-Control vector containing luciferase reporter under control of SV40 promoter served as a control. Each transfection was repeated three times and the luciferase activity was measured twice for each sample.

\section{Preparation of nuclear extracts}

Five-day old, adult female mosquitoes were dissected on a clean slide in a drop of ice-cold sterile phosphate-buffered saline (PBS) pH 7.4 under a dissecting microscope. The midgut, head, legs and wings were removed and the body tissue of 100 to 200 mosquitoes from both R and S strains were pooled and stored under liquid nitrogen until required. The tissues were ground to homogeneity in a minimum volume of PBS, with a battery-driven handheld homogenizer using sterile grinder tips and processed for nuclear protein extraction according to the protocol of Lin et al., (2004) [45] with minor modifications. Briefly, homogenized tissue was solubilized in buffer A $(10 \mathrm{mM}$ HEPES, $10 \mathrm{mM} \mathrm{KCl}, 1 \mathrm{mM}$ EDTA, $1 \mathrm{mM}$ dithiothreitol, $0.5 \mathrm{mM}$ phenylmethylsulfonyl fluoride, $0.1 \mathrm{mM} \mathrm{Na}_{3} \mathrm{VO}_{4}$, $10 \%$ glycerol and $1 \mu \mathrm{g} / \mathrm{ml}$ of aprotinin, pepstatin, and leupeptin (pH 7.9) for $30 \mathrm{~min}$ at $4^{\circ} \mathrm{C}$ by gentle rocking on a nutator and centrifuged at $12,000 \times \mathrm{g}$ at $4^{\circ} \mathrm{C}$ for $1 \mathrm{~min}$. The supernatant containing the cytoplasmic proteins was discarded and the pellet was then extracted with buffer $\mathrm{B}$ (20 mM HEPES, $350 \mathrm{mM} \mathrm{NaCl}, 10 \mathrm{mM} \mathrm{KCl}, 1 \mathrm{mM}$ EDTA, $1 \mathrm{mM}$ dithiothreitol, $1 \mathrm{mM}$ phenylmethylsulfonyl fluoride, $0.1 \mathrm{mM} \mathrm{Na}_{3} \mathrm{VO}_{4}, 0.2 \%$ Nonidet P- $40,10 \%$ glycerol, $1 \mu \mathrm{g} / \mathrm{ml}$ aprotinin, pepstatin and leupeptin, $\mathrm{pH}$ 7.9) at $4^{\circ} \mathrm{C}$ for one hour by gentle rocking on a nutator as described above. The extracts were centrifuged at 13,000 $\times$ $g$ at $4{ }^{\circ} \mathrm{C}$ for 5 minutes and the supernatants (nuclear extract) were snap frozen in liquid $\mathrm{N}_{2}$ and stored at $-70^{\circ} \mathrm{C}$ for subsequent use in EMSAs. All the protein concentrations were estimated by the method of Bradford (1976) [46].

\section{Electrophoretic mobility shift assay (EMSA)}

Primers were designed to encompass the 400 bp upstream region (-702 to $-302 \mathrm{bp}$ ) of acsp30 that was responsible for difference in promoter strength in the two strains. Three different fragments (100 bp, $188 \mathrm{bp}$ and $400 \mathrm{bp}$ ) from upstream regions of $\mathrm{R}$ and $\mathrm{S}$ strains were selected for EMSAs. The DNA probes for EMSAs were prepared by amplifying and radioactively labeling the fragments by PCR in a $50 \mu \mathrm{l}$ reaction mixture. The reaction consisted of 10 ng template (upstream regions from $R$ and $S$ ), $10 \mathrm{pmol}$ of each primer, $100 \mu \mathrm{M}$ dNTPs, $10 \mu \mathrm{l} \alpha$-32P-dCTP (10 $\mathrm{mCi} / \mathrm{ml}$, $3000 \mathrm{Ci} / \mathrm{mmol}$, PerkinElmer, USA), and 2.5 units of Taq DNA polymerase (Clontech, USA). The PCR included 32 cycles of $30 \mathrm{~s}$ denaturation at $94^{\circ} \mathrm{C}, 30 \mathrm{~s}$ annealing at $52^{\circ} \mathrm{C}$ and $30 \mathrm{~s}$ extension at $72^{\circ} \mathrm{C}$, followed by a final extension of $5 \mathrm{~min}$ at $72^{\circ} \mathrm{C}$. Labeled probes were purified using a QIAquick nucleotide removal kit (Qiagen $\mathrm{GmbH}$ ) and the counts were checked by scintillation counter (Beckman, USA).

The probes were named based on the size of the amplified DNA fragment. In the R strain, R100 probe (-702 and -602 bp) was amplified using RefUp702For (5'-CAA TGA CGC GTT AAG CCT GAT-3') and RefUp602Rev (5'-GAT CGC CGT CGT CCA TCA ACA-3') primers; R188 probe (-702 and -514 bp) by RefUp702For and RefUp514Rev (5'-TTC ATG TGG TTT CAT GAT TTA TTA-3') primers; R400 probe (-702 and -302 bp) by RefUp702For and RefUp302Rev (5'-CTT TCG AAT GAC GCC AGG T-3') primers. Similarly for the $S$ strain, $S 100$ probe (-702 and $-602 \mathrm{bp}$ ) was amplified using SusUp702For (5'-TCC TGA TCA ACT ATA TGG GTT CCT-3') and SusUp602Rev (5'-CTA TTG AAA GAA TCA ATT TGC TAA-3') primers; S188 probe (-702 and 514 bp) by SusUp702For and SusUp514Rev (5'-TTC ATA TCT CAT CAT TTA TTA AAA ATT-3') primers; S400 probe (-702 and -302 bp) by SusUp702For and SusUp302Rev (5'-ATT TCG TAA TGA CGG CCA GGT-3') primers. EMSAs were performed using 10 to $20 \mu \mathrm{g}$ of nuclear extract from either $\mathrm{R}$ or $\mathrm{S}$ and incubated at $37^{\circ} \mathrm{C}$ for 25 minutes with ${ }^{32}$ P-labeled probes (30,000 c.p.m. per reaction) and $2 \mu \mathrm{g}$ of poly (dI-dC) (Sigma). The binding buffer contained 20 mM HEPES (pH 7.9), $1 \mathrm{mM}$ dithiothreitol, $1 \mathrm{mM}$ EDTA (pH 8.0), $1.5 \mathrm{mM} \mathrm{MgCl}_{2}$ and $4 \%$ glycerol, in a final volume of $20 \mu \mathrm{l}$. For competition experiments, a 100-fold molar excess of unlabeled probe was pre-incubated in the reaction mixture at $37^{\circ} \mathrm{C}$ for $10 \mathrm{~min}$ before labeled probe was added. The DNA-protein complex was resolved on a $2 \%$ agarose gel (pre-run for $1 \mathrm{~h}$ at $4^{\circ} \mathrm{C}$ ) in $0.5 \times$ Tris-borate EDTA (TBE) buffer at $200 \mathrm{~V}$ at $4^{\circ} \mathrm{C}$. Gels were dried and exposed for autoradiography. Scanning was performed using the Typhoon 9210, Variable Mode Imager from Amersham Biosciences, USA. The intensities of the signals were quantified by the Image Analysis Software, ImageQuant TL (Amersham Biosciences) and represented as the percentage ratio of $S$ to $R$ signal intensities. The accession number of the serine protease gene is [GeneBank:AY995188].

\section{Authors' contributions}

JR carried out real-time PCR, transfection studies in cell culture and EMSAs. NA carried out isolation and cloning of serine protease gene and its upstream regulatory sequences. Both, JR and NA, have also been involved in drafting the manuscript. AS carried out mosquito rearing and blood feeding experiments. PM performed EMSA experiments and gave important input in analysis and interpretation of data. TA provided the susceptible and 
refractory strains of A. culicifacies and was involved in dissections of mosquitoes, collection of body tissue, preparation of nuclear extracts and participated in the design of the study. VSC gave final approval of the version to be published. VSC and RKB conceived of the study, participated in its design and coordination and also revised the manuscript critically. All authors read and approved the final manuscript.

\section{Acknowledgements}

We gratefully acknowledge the financial support provided by both, Indian Council for Medical Research (ICMR) and Department of Science and Technology (DST), Government of India during this course of study. We also acknowledge help rendered by Dr. Kokali Ghosh at National Institute of Immunology, New Delhi, for access to luminometer. We acknowledge the editorial assistance of the NIH Fellows Editorial Board.

\section{References}

I. Sharma VP: Current scenario of malaria in India. Parasitologia 1999, $41: 349-353$

2. Beernsten BT, James AA, Christensen BM: Genetics of mosquito vector competence. Microbiol Mol Biol Rev 2000, 64:II5-137.

3. Collins FH, Sakai RK, Vernick KD, Paskewitz S, Seeley DC, Miller LH, Collins WE, Campbell CC, Gwadz RW: Genetic selection of a Plasmodium-refractory strain of the malaria vector Anopheles gambiae. Science 1986, 234:607-6I0.

4. Schwartz A, Koella JC: Melanization of Plasmodium falciparum and C-25 sephadex beads by field-caught Anopheles gambiae (Diptera, Culicidae) from southern Tanzania. J Med Entomol 2002, 39:84-88.

5. Blandin S, Shiao S-H, Moita LF, Janse CJ, Waters AP, Kafatos FC Levashina EA: Complement-like protein TEPI is a determinant of vectorial capacity in the malaria vector Anopheles gambiae. Cell 2004, I 16:66 I-670.

6. Paskewitz SM, Brown MR, Lea AO, Collins FH: Ultrastructure of the encapsulation of Plasmodium cynomolgi (B strain) on the midgut of a refractory strain of Anopheles gambiae. J Parasitol 1988, 74:432-439.

7. Kaur S, Adak T, Singh OP: Susceptibility of species A, B, C of Anopheles culicifacies complex to Plasmodium yoelii and Plasmodium vinckei petteri infection. J Parasitol 2000, 86: I345-1348.

8. Adak T, Singh OP, Nanda N, Sharma VP, Subbarao SK: Malariarefractory Anopheles culicifacies. Trop Med Intl Health 2006 , 2:I-7.

9. Nappi AJ, Sugumaran M: Some biochemical aspects of eumelanin formation in insect immunity. In Insect Immunity Edited by: Pathak JPN. Oxford: IBM Publishing; 1993:|3|-I48.

10. Kumar S, Christophides GK, Cantera R, Charles B, Han YS, Meister $S$, Dimopoulos G, Kafatos FC, Barillas-Mury C: The role of reactive oxygen species on Plasmodium melanotic encapsulation in Anopheles gambiae. Proc Natl Acad Sci USA 2003, 100:14|39-|4| 44

II. Ashida M, Brey PT: Role of the integument in insect defense, pro-phenol oxidase cascade in the cuticular matrix. Proc Nat Acad Sci USA 1995, 92: 10698-10702.

12. Soderhall K, Cerenius $L$ : Role of the prophenoloxidase-activating system in invertebrate immunity. Curr Opin Immunol 1998, 10:3-28.

13. Dimopoulos G, Richman A, Muller H-M, Kafatos FC: Molecular immune responses of the mosquito Anopheles gambiae to bacteria and malaria parasites. Proc Natl Acad Sci USA 1997. 94:II508-11513

14. Dimopoulos G, Seeley D, Wolf A, Kafatos FC: Malaria infection of the mosquito Anopheles gambiae activates immune-responsive genes during critical transition stages of the parasite life cycle. EMBO J 1998, 17:6||5-6|23.

15. Paskewitz SM, Reese-Stardy S, Gorman MJ: An easter-like serine protease from Anopheles gambiae exhibits changes in transcript abundance following immune challenge. Insect Mol Biol 1999, 8:329-337.
16. Gorman MJ, Andreeva OV, Paskewitz SM: Molecular characterization of five serine protease genes cloned from Anopheles gambiae hemolymph. Insect Biochem Mol Biol 2000, 30:35-46.

17. Gorman MJ, Paskewitz SM: Serine proteases as mediators of mosquito immune responses. Insect Biochem Mol Biol 200I, $31: 257-262$.

18. Volz J, Osta MA, Kafatos FC, Muller H-M: The roles of two clip domain seine proteases in innate immune responses of the malaria vector Anopheles gambiae. I Biol Chem 2005, 280:40|6|-40|68.

19. Paskewitz SM, Andreev O, Shi L: Gene silencing of serien proteases affects melanization of sephadex beads in Anopheles gambiae. Insect Biochem Mol Biol 2006, 36:70I-7I I.

20. Volz J, Muller H-M, Zdanowicz A, Kafatos FC, Osta MA: A genetic module regulates the melanization response of Anopheles to Plasmodium. Cell Microbiol 2006, 8: I392-I 405.

21. Han Y-S, Salazar CE, Reese-Stardy, Cornel A, Gorman MJ, Collins FH, Paskewitz SM: Cloning and characterization of a serine protease from human malaria vector, Anopheles gambiae. Insect Mol Biol 1997, 6:385-395.

22. Muharsini S, Dalrymple B, Vuocolo T, Hamilton S, Willadsen P, Wijffels G: Biochemical and molecular characterization of serine proteases from larvae of Chrysomya bezziana, the Old World Screwworm fly. Insect Biochem Mol Biol 200I, 31:1029-1040.

23. Ligoxygakis P, Pelte N, Hoffmann JA, Reichhart JM: Activation of Drosophila Toll during fungal infection by a blood serine protease. Science 2002, 297: I I4-II6

24. Söderhäll K, Iwanaga S, Vasta G: New Directions in Invertebrate Immunology New Jersey: SOS publications; 1996.

25. Kanost MR, Jiang H, Wang Y, Yu XQ, Ma C, Zhu Y: Hemolymph proteinases in immune responses of Manduca sexta. Adv Exp Med Biol 200I, 484:319-328.

26. Zheng L, Cornel AJ, Wang R, Erfle H, Voss H, Ansorge W, Kafatos FC, Collins FH: Quantitative trait loci for refractoriness of Anopheles gambiae to Plasmodium cynomolgi B. Science 1997, 276:425-428.

27. Cherbas $L$, Cherbas $P$ : The arthropod initiator: the cap site consensus plays an important role in transcription. Insect Biochem Mol Biol 1993, 23:8I-90.

28. Quandt K, French K, Karas H, Wingender E, Werner T: MatInd and Matlnspector: new fast and versatile tools for detection of consensus matches in nucleotide sequence data. Nucleic Acids Res 1995, 23:4878-4884.

29. Eggleston P, Lu W, Zhao Y: Genomic organization and immune regulation of the defensin gene from the mosquito, Anopheles gambiae. Insect Mol Biol 2000, 9:48I-490.

30. Edwards MJ, Severson DW, Hagedorn HH: Vitelline envelope genes of the yellow fever mosquito, Aedes aegypti. Insect Biochem Mol Biol 1998, 28:915-925.

31. Vernick KD, Fujioka H, Seeley DC, Tandler B, Aikawa M, Miller LH: Plasmodium gallinaceum: a refractory mechanism of ookinete killing in the mosquito, Anopheles gambiae. Exp Parasitol 1995, 80:583-595.

32. Jiang $H$, Kanost MR: The clip-domain family of serine proteinases in arthropods. Insect Biochem Mol Biol 2000, 30:95-105.

33. Morisato $D$, Anderson KV: Signaling pathways that establish the dorsal-ventral pattern of the Drosophila embryo. Annu Rev Genet 1995, 29:37I-399.

34. Barillas-Mury C, Charlesworth A, Gross I, Richman A, Hoffmann JA Kafatos FC: Immune factor Gambifl, a new rel family member from the human malaria vector, Anopheles gambiae. EMBO 1996, 15:469|-4701.

35. Cho WL, Fu TF, Chiou JY, Chen CC: Molecular characterization of a Defensin gene from the mosquito, Aedes aegypti. Insect Biochem Mol Biol | 997, 27:35I-358.

36. Zheng $L X$, Zheng L: Genomic organization and regulation of three Cercopin genes in Anopheles gambiae. Insect Mol Biol 2002, I I:517-524.

37. Sun D, Fallon AM: Characterization of genomic DNA encoding cecropins from an Aedes albopictus mosquito cell line. Insect Mol Biol 2002, I I:2I-30.

38. Ahmed A, Martin D, Manetti AGO, Han SJ, Lee WJ, Mathiopoulos KD, Muller H-M, Kafatos FC, Raikhel A, Brey PT: Genomic structure and ecdysone regulation of the prophenoloxidase $I$ gene in the malaria vector Anopheles gambiae. Proc Natl Acad Sci USA 1999, 96:14795-14800. 
39. Adak T, Kaur S, Singh OP: Comparative susceptibility of different members of the Anopheles culicifacies complex to Plasmodium vivax. Trans Roy Soc Trop Med Hyg 1999, 93:573-577.

40. Henry JM, Raina AK, Ridgway P: Isolation of high molecular weight DNA from insects. Anal Biochem 1990, 185: I47-150.

41. Landau I, Boulard Y: Life cycles and morphology. In Rodent malaria Edited by: Killick-Kendrick R, Peters W. London: Academic Press; 1978:53-84.

42. Singh J: JSB stain - a review. Ind J Malarial I956, 24:9-I5.

43. Livak KJ: ABI Prims 7700 Sequencer detection system, User bulletin 2. PE Applied Biosystems 1997: I-36.

44. Mishra RN, Singla-Pareek SL, Nair S, Sopory SK, Reddy MK: Directional genome walking using PCR. Biotechniques 2002, 33: I-4.

45. Lin CC, Chou CM, Hsu YL, Lien JC, Wang YM, Chen ST, Tsai SC, Hsiao PW, Huang CJ: Characterization of two mosquito STATs, AaSTAT and CtSTAT. Differential regulation of tyrosine phosphorylation and DNA binding activity by lipopolysaccharide treatment and by Japanese encephalitis virus infection. J Biol Chem 2004, 279:3308-33I7.

46. Bradford MM: A rapid and sensitive method for the quantitation of microgram quantities of protein utilizing the principle of protein-dye binding. Anal Biochem 1976, 72:248-254.

47. The subcellular localization prediction tool [http://
[ www.psort.org]

48. UniProt (Universal Protein Resource) [http://www.pir.uni prot.org]

49. The promoter prediction tool [http://www.fruitfly.org]

50. The Primer 3 web-based tool [http://www.genome.wi.mit.edu/ cgi-bin/primer/primer3 www.cgi]

51. The MatInspector program [http://www.genomatix.de]

52. EMBOSS alignment program [http://www.ebi.ac.uk/emboss/ align]

Publish with Bio Med Central and every scientist can read your work free of charge

"BioMed Central will be the most significant development for disseminating the results of biomedical research in our lifetime. "

Sir Paul Nurse, Cancer Research UK

Your research papers will be:

- available free of charge to the entire biomedical community

- peer reviewed and published immediately upon acceptance

- cited in PubMed and archived on PubMed Central

- yours - you keep the copyright

Submit your manuscript here:

http://www.biomedcentral.com/info/publishing_adv.asp
BioMedcentral 